\title{
Analysis of Stresses in Metal Sheathed Thermocouples in High-Temperature, Hypersonic Flows
}

\author{
Sean William Powers \\ Thesis submitted to the Faculty of the \\ Virginia Polytechnic Institute and State University \\ in partial fulfillment of the requirements for the degree of
}

Master of Science

in

Aerospace Engineering

Joseph A. Schetz, Co-chair

K. Todd Lowe, Co-chair

Rakesh K. Kapania

April 17, 2020

Blacksburg, Virginia

Keywords: Thermocouples, Survivability, Low-Order Modeling, Lamé's Stresses, Hypersonics, High-Temperature 


\title{
Analysis of Stresses in Metal Sheathed Thermocouples in High-Temperature, Hypersonic Flows
}

\author{
Sean William Powers
}

(ABSTRACT)

Flow temperature sensing remains important for many hypersonic aerodynamics and propulsion applications. Flight test applications, in particular, demand robust and accurate sensing, making thermocouple sensors attractive. Even for these extremely well-developed sensors, the prediction of stresses (hoop, radial, and axial) within thermocouple sheaths for custom-configured probes remains a topic of great concern for ensuring adequate lifetime of sensors. In contemporary practice, high-fidelity simulations must be run to prove if a new design will work at all, albeit at significant time and expense. Given the time and money it takes to run high-fidelity simulations, rapid optimization of sensor configurations is often impossible, or at a minimum, impractical. The developments presented in this Thesis address the need for hypersonic flow temperature sensor structural predictions which are compatible with rapid design iteration. The derivation and implementation of a new analytical, loworder model to predict stresses (hoop, radial, and axial) within the sheath of a thermocouple are provided. The analytical model is compared to high-fidelity ANSYS mechanical simulations as well as simplified experimental data. The predictions using the newly developed structural low-order model are in excellent agreement with the numerically simulated results and experimental results with an absolute maximum percent error of approximately $4 \%$ and 9.5\%, respectively, thus validating the model. A MATLAB GUI composed of the combination of a thermal low-order model outlined in additional references [1] through [6] and the new structural low-order model for thermocouples was developed. This code is capable of solving a highly generalized version of the 1-D pin fin equation, allowing for the solution of the temperature distribution in a sensor taking into account conduction, convection, and radiation heat transfer which is not possible with other existing analytical solutions. This temperature distribution is then used in the analytical structural low-order model. This combination allows for the thermal and structural performance of a thermocouple to be found analytically and compared quickly with other designs. 


\title{
Analysis of Stresses in Metal Sheathed Thermocouples in High-Temperature, Hypersonic Flows
}

\author{
Sean William Powers \\ (GENERAL AUDIENCE ABSTRACT)
}

Thermocouples are a device for measuring temperature, consisting of two wires of different metals connected at two different points. This configuration produces a temperaturedependent voltage as a result of the thermoelectric effect. Preexisting curves are used to relate the voltage to temperature. Thermocouples are extensively used in high-temperature high-stress environments such as in rockets, jet engines, or any high-corrosive environment. Accurately predicting the stresses within the sheath of a metal-clad thermocouple in extreme conditions is required for many research areas including hypersonic aerodynamics and various propulsion applications. Even for these extremely well-developed and widely used sensors, the accurate prediction of stresses within the metal sheath remains a topic of great concern for ensuring the sensor's survivability in these extreme conditions. Current engineering practice is to use high-fidelity numerical simulations (Finite Element Analysis) to predict the stresses within the sheath. Perhaps the biggest drawback to this approach is the time it takes to model, mesh, and set-up these simulations. Comparative studies between different designs using numerical simulations are almost impossible due to the time requirement. This Thesis will present an analytically derived quasi-3D solution to find the stresses within the sheath. These equations were implemented into a low-order model that can handle varying temperature, geometry, and material inputs. This model was validated against both high-fidelity numerical simulations (ANSYS Mechanical) and a simplified experiment. The predictions using this newly developed structural low-order model are in excellent agreement with the numerically simulated results and experimental results. 


\section{Acknowledgments}

Many individuals deserve my acknowledgment and thanks for their contribution and support throughout my time here at Virginia Tech. I would like to start with my co-advisor Dr. Joseph Schetz. It was because of his trust and unwavering support in me as a sophomore in my undergraduate studies that I have been afforded such amazing opportunities such as attending graduate school and so many more that I have yet to realize. Secondly, I would like to extend my greatest gratitude to Dr. Todd Lowe for allowing me to join a worldclass research team as a sophomore. This appointment allowed me the opportunity to learn from the best and brightest minds that Virginia Tech has to offer. I would like to thank both of these gentlemen for supporting and shaping me into the best engineer, student, and researcher that I could be. None of this would have been possible without their trust in me. I would also like to thank Dr. Rakesh Kapania for teaching and guiding me in the use of fundamental techniques seen in this Thesis. Without his continual guidance and willingness to answer my questions, this would not have been possible.

I would like to thank Pratt and Whitney for funding this research and for providing excellent opportunities through the continued partnership with Virginia Tech. I would also like to acknowledge and thank Ian Agoos, Tyler Englerth, Dr. Charles Haldeman, and Andy Consiglio at Pratt and Whitney for their insightful technical discussions and project feedback. It is your feedback and support that helped progressed this project and myself as an engineer.

Next, I would like to acknowledge the necessary support of my friends, many of whom I have met during my years at Virginia Tech as an undergrad and now a graduate student. I have enjoyed our time together outside of work and will always cherish our life-long friendship. I would also like to thank my team members Dr. Tyler Vincent and Reuben Quickel for helping me grow as an undergrad and then providing valuable assistance as a graduate student.

Finally, I would like to dedicate this Thesis to my family who has always supported me in my many endeavors. To my mom and step-dad, Angie and Jason Smith, my dad, Todd Powers, my brother, Colin Smith, and my sister, Bekah Smith, I love you.

Sean W. Powers 


\section{Attributions}

Several professors and colleagues contributed to the research and writing contained in this Thesis. A brief description of their contributions if included below:

Dr. Joseph A. Schetz and Dr. K. Todd Lowe are co-advisors and committee chairs for this research. Both provided extensive guidance on the research plan, secured research funding, and provided editorial revisions and advice on the analysis presented in this Thesis.

Dr. Rakesh K. Kapania is an advisor for this research. He provided editorial revisions and advice on all the analyses presented in this Thesis.

Dr. Charles Haldeman and Andy Consiglio are technical fellows at Pratt and Whitney. Both provided extensive guidance on the research plan.

Ian Agoos and Tyler Englerth are engineers in the Systems Engineering and Validation group at Pratt and Whitney. Both provided extensive guidance on the research plan and facilitated weekly technical discussions. 


\section{Contents}

List of Figures $\quad$ viii

List of Tables $\quad$ x

1 Introduction $\quad 1$

1.1 Thesis Structure and Contents . . . . . . . . . . . . . . . . 2

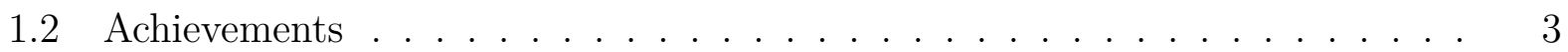

2 Analysis of Stresses in Metal Sheathed Thermocouples in High-Temperature, $\begin{array}{ll}\text { Hypersonic Flows } & 4\end{array}$

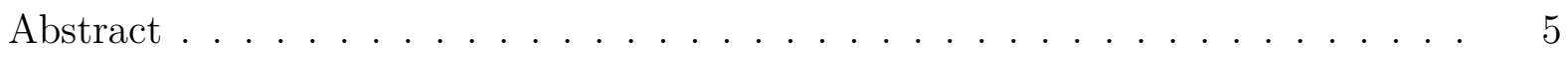

Nomenclature ............................ 5

2.1 Introduction . . . . . . . . . . . . . . . . . . . 6

2.2 Analysis ................................ 7

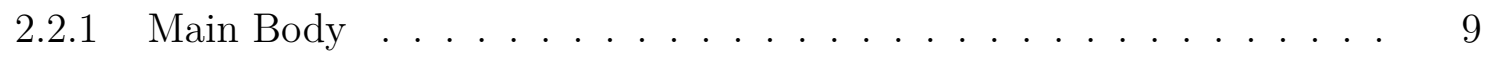

2.2.2 Clamped Base . . . . . . . . . . . . . . . 12

2.2 .3 Transition Zone . . . . . . . . . . . . . . . . . . 13

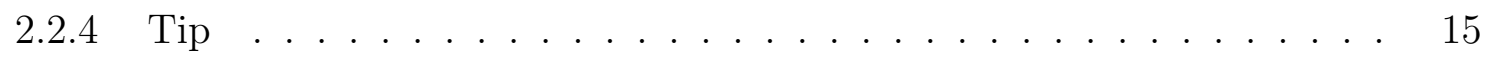

2.3 Comparison of Analytical and Numerical Results . . . . . . . . . . . . 17

2.3.1 ANSYS Mechanical Modeling and Statistics . . . . . . . . . . 19

2.3.2 High-Fidelity Simulation and Low-Order Model Results Comparison 20

2.4 Comparison of Analytical and Experimental Results . . . . . . . . . . . . . 24 
2.5 Conclusions . . . . . . . . . . . . . . . . . . . . . . . . . . . . . . 29

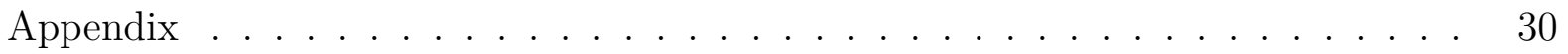

Acknowledgments . . . . . . . . . . . . . . . . . . 33

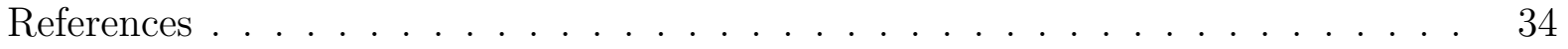

3 Conclusions and Outlook 35

3.1 Conclusions . . . . . . . . . . . . . . . . . . . . . 35

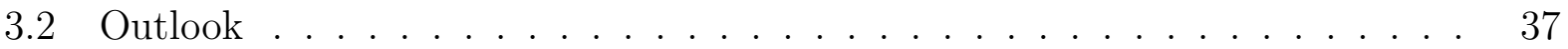

$\begin{array}{ll}\text { Bibliography } & 39\end{array}$ 


\section{List of Figures}

2.1 Thermocouple Section and Junction Classifications _ . . . . . . . . . 6

2.2 Thermocouple Geometry Layout _ . . . . . . . . . . . . . . 7

2.3 Thermocouple Configuration and Area Averaging Simplification Schemes . . 8

2.4 Thermocouple Simplification Study Results (Simulation Contours) . . . . . 8

2.5 Thermocouple Simplification Study Results (Line Stress Comparison) . . . . 9

2.6 Application of the No-Slip Condition . . . . . . . . . . . . . . . 10

2.7 Radii and Pressures Acting on Each Body . . . . . . . . . . . . . . 11

2.8 Thermocouple Base Geometry and Mounting Structure . . . . . . . . . . . 12

2.9 Base Simplification Technique . . . . . . . . . . . . . . . . . . . . . 13

2.10 Five-Body System with Four Unknown Pressures . . . . . . . . . . . . . 13

2.11 Transition Zone of the Thermocouple . . . . . . . . . . . . . . . . . 14

2.12 The Three-Body System with Two Unknown Pressures . . . . . . . . . . . 15

2.13 Spherical and Cylindrical Axis Comparison . . . . . . . . . . . . . 16

2.14 Tip Equation Averaging using Angle Weighted Averaging . . . . . . . . . 16

2.15 3D Model made in ANSYS Mechanical . . . . . . . . . . . . . . . . 18

2.16 Equivalent Model Used with the Analytical Equations in the Low-Order Model 18

2.17 3D Meshing in ANSYS Mechanical . . . . . . . . . . . . . . . 19

2.18 Displacement Boundary Condition _... . . . . . . . . . . . 20

2.19 High-Fidelity Comparison with the Simplified Model in ANSYS . . . . . . 21

2.20 Stress Results from a Cross-Sectional Cut in ANSYS and the Low-Order Model 21 
2.21 Temperature Profile and Stress Value Comparison Between ANSYS Mechanical and the Analytical Low-Order Model in the Sheath . . . . . . . . . . . 22

2.22 Clamped Base and Transition Zone Stress Results . . . . . . . . . . . . . . 22

2.23 Main Body Stress Result Comparisons . . . . . . . . . . . . . . . . . . 23

2.24 Tip Stress Results . . . . . . . . . . . . . . . . . . . 24

2.25 Exaggerated Tip Geometry Change due to Thermal Expansion . . . . . . . . 24

2.26 Experimental Rods Vs. Typical Thermocouple Configuration . . . . . . . . . 25

2.27 Strain Gauge Placement . . . . . . . . . . . . . . . . . . . 25

2.28 Experimental Setup . . . . . . . . . . . . . . . . . . 26

2.29 Raw Transient Voltage Data Vs. Steady-State Voltage Data . . . . . . . 26

2.30 Strain Gauge Wire Setup . . . . . . . . . . . . . . . . . . . 27

2.31 Gauge Factor Correction Variation with Temperature . . . . . . . . . . 27

2.32 Curvature Strain Correction Variation with Temperature . . . . . . . . . 28

2.33 Experimental Results Comparison with Analytical Predictions . . . . . . . 29

3.1 Structural Dynamics of Extreme Environment Thermocouples. . . . . . . . . 37

3.2 Visual Depiction of Sheath Failure Theory. . . . . . . . . . . . . . . . . 38 


\section{List of Tables}

2.1 ANSYS Mechanical Meshing Statistics _ . . . . . . . . . . . . . . 19

2.2 Magnesium Oxide Material Properties _. . . . . . . . . . . . . . . 30

2.3 Inconel 600 Material Properties . . . . . . . . . . . . . . . . . . . 31

2.4 Platinum-Rhodium $30 \%$ Material Properties . . . . . . . . . . . . . . 31

2.5 Platinum-Rhodium $20 \%$ Material Properties . . . . . . . . . . . . . . . 32

2.6 Platinum-Rhodium $10 \%$ Material Properties . . . . . . . . . . . . . . . . 32

2.7 Airfoil Profile Coordinates . . . . . . . . . . . . . . . . . . . . . 33 


\section{Chapter 1}

\section{Introduction}

Sheathed thermocouples allow for temperature sensing of flows involving heat transfer and/or chemical reactions. Thermocouples provide a robust measurement system that is necessary for fight tests and advanced development applications and are highly regarded for their reliability in high-stress environments. However, there remains a major need for a quick and accurate way to predict the stresses in a metal-clad thermocouple packed with ceramic material at elevated temperatures. An accurate low-order model for predicting the thermal performance of such a thermocouple has already been published in additional references [1] through [6].

The desire to have high-survivability thermocouples at elevated temperatures stems from the frequent failure of these probes during testing. These failures lead to the loss of time, money, and valuable data. These stresses can be predicted using high-fidelity codes at very high cost and time. To the authors' knowledge, no accurate low-order model has been produced to predict the stresses within the sheath of a thermocouple. Engineering research practice has often strayed away from an analytical model in favor of a numerical model for use in capturing 3D effects, especially when involving complicated shapes where common analytical equations break down and are not accurate. In the current case, the mating of a cylindrical body to a spherical tip that is filled with potting and wires culminates in complex interactions between the various components which cannot be easily handled by traditional analytical equations. The present study seeks to understand, derive, and implement quasi3D analytical equations to create a low-order model for these complex interactions ultimately resulting in an analytical model for the stresses in the sheath of a thermocouple. 


\subsection{Thesis Structure and Contents}

This Thesis is divided into the following chapters:

Chapter 1 provides a general introduction to the topic and overview of the Thesis.

Chapter 2 is the manuscript, "Analysis of Stresses in Metal Sheathed Thermocouples in High-Temperature, Hypersonic Flows", to be published in the conference proceedings of the 2020 AIAA Aviation conference. This paper provides a deeper understanding of the structural stresses present in thermocouples by developing an analytical model. This was accomplished in part by using high-fidelity structural modeling within ANSYS Mechanical to compare to the analytical models being developed. The analytical models rely on a quasi3D extension of Lamès 2D circular cylinder and spherical equations. Deriving the quasi-3D equations led to the discovery of the underlying causes of major stresses within the sheath of a high-temperature thermocouple. If the thermal expansion coefficient of the inner ceramic potting is greater than that of the metal sheath then large stresses are built up in the sheath as the thermocouple thermally expands. If the thermal expansion coefficient of the sheath is larger than that of the potting then little stress results from thermal expansion. This statement was then tested against widely used thermocouple types, and it was found that all thermocouples meant for very high-temperature flows suffer from the former, while low-temperature probes are indicative of the latter condition. Specifically in this paper, a Platinum-Rhodium sheath with Magnesium-Oxide potting was studied for hypersonic flight at 30,000 feet flying at Mach 5. This means the tip of the thermocouple is roughly at $2000^{\circ} \mathrm{F}$, while the base is roughly at $1500^{\circ} \mathrm{F}$. This test case was run through both ANSYS and the developed analytical model. Taking the ANSYS results to be true, the new analytical model had approximately $2 \%$ error in the tip, $1 \%$ error in the middle section, and $6 \%$ error in the base. Lastly, the analytical model was tested against a simplified experiment with the length to diameter ratio over ten. The model showed approximately $7 \%$ error and $9 \%$ error in the circumferential (hoop) and axial directions, respectively. In an extreme case, when the length to diameter ratio was small, which violates some assumptions made in the loworder model, the model showed approximately $4 \%$ error and $42 \%$ error in the circumferential and axial directions, respectively. It was shown that hoop stress has the highest impact in 
comparison with both radial and axial stress.

Chapter 3 concludes the overall research efforts and provides an outlook on future work.

Formatting chapter 2 contents and citation style deviate due to the different publication guidelines of AIAA Aviation.

\subsection{Achievements}

Key outcomes of the present research include:

- Development and verification of a new low-order analytic structural stress model for thermocouples designed to account for various flow conditions, temperature profiles, material selection, and varying geometry.

- A MATLAB implementation of the aforementioned structural low-order model.

- A MATLAB GUI composed of the combination of a thermal low-order model outlined in additional references [1] through [6] and the new structural low-order model for thermocouples was developed. This code is capable of solving a highly generalized version of the 1-D pin fin equation, allowing for the solution of the temperature distribution in a sensor taking into account conduction, convection, and radiation heat transfer which is not possible with other existing analytical solutions. This temperature distribution is then used in the analytical structural low-order model. This combination allows for the thermal and structural performance of a thermocouple to be found analytically and compared quickly with other designs. 


\section{Chapter 2}

\section{Analysis of Stresses in Metal Sheathed Thermocouples in High-Temperature, Hypersonic Flows}

The contents of this chapter have been accepted into the AIAA Aviation conference as a conference paper (Sean W. Powers, Joseph A. Schetz, K. Todd Lowe, and Rakesh K. Kapania. "Analysis of Stresses in Metal Sheathed Thermocouples in High-Temperature, Hypersonic Flows"). 


\title{
Analysis of Stresses in Metal Sheathed Thermocouples in High-Temperature, Hypersonic Flows
}

\author{
Sean W. Powers*, Joseph A. Schetz ${ }^{\dagger}$, K. Todd Lowe ${ }^{\ddagger}$, Rakesh K. Kapania $^{\S}$ \\ Virginia Polytechnic Institute and State University, Blacksburg, VA, 24060, United States
}

Near-wall flow temperature sensing remains important for many hypersonic aerodynamics and propulsion applications. Flight test applications, in particular, demand robust and accurate sensing, making thermocouple sensors attractive. Even for these extremely well-developed sensors, the prediction of stresses within thermocouple sheaths for custom-configured probes remains a topic of great concern for ensuring adequate lifetime of sensors. In contemporary practice, high-fidelity simulations must be run to prove if a new design will work at all, albeit at significant time and expense. Given the time and money it takes to run high-fidelity simulations, rapid optimization of sensor configurations is often impossible, or at minimum, impractical. The developments presented in this paper address the need for hypersonic flow temperature sensor structural predictions which are compatible with rapid design iteration. The derivation and implementation of a new analytical, low-order model to predict stresses within the sheath of a thermocouple are provided. The analytical model is compared to high-fidelity ANSYS mechanical simulations as well as simplified experimental data. The predictions using the newly developed structural low-order model are in excellent agreement with the numerically simulated results and experimental results with an absolute maximum percent error of approximately $4 \%$ and $9.5 \%$, respectively, thus validating the model.

Symbols

\section{Nomenclature}

$\begin{array}{ll}\alpha & =\text { Thermal Expansion Coefficient } \\ p & =\text { Pressure } \\ T & =\text { Radial Location } \\ r & =\text { Poisson's Ratio } \\ v & =\text { Young's Modulus } \\ E & =\text { Cross-Sectional Area } \\ A & =\text { Lamé's First Parameter } \\ \lambda & =\text { Lamé's Second Parameter } \\ \mu & =\text { Stress in the Radial Direction (Cylindrical Coordinates) } \\ \sigma_{r r_{C}} & =\text { Stress in the Theta Direction (Cylindrical Coordinates) } \\ \sigma_{\theta \theta_{C}} & =\text { Stress in the Axial Direction (Cylindrical Coordinates) } \\ \sigma_{z z_{C}} & =\text { Stress in the Radial Direction (Spherical Coordinates) } \\ \sigma_{r r_{S}} & =\text { Stress in the Theta Direction (Spherical Coordinates) } \\ \sigma_{\theta \theta_{S}} & =\text { Stress in the Phi Direction (Spherical Coordinates) } \\ \sigma_{\phi \phi_{S}} & =\text { Gauge factor correction with temperature } \\ \text { Gauge } e_{F a c} & =\text { Scaling base gauge factor } \\ F^{*} & =\text { Actual gauge factor at room temperature } \\ F_{G a u g e} & =\text { Percentage of gauge factor change with room temperature } \\ \Delta F(\%) & =\text { Strain correction for a curved body } \\ \epsilon_{C u r v} & =\text { radius of curvature } \\ R & \end{array}$

*Graduate Research Assistant, Kevin T. Crofton Department of Aerospace and Ocean Engineering, 460 Old Turner St, Blacksburg, VA 24060, AIAA Student Member.

${ }^{\dagger}$ Fred D. Durham Endowed Chair, Kevin T. Crofton Department of Aerospace and Ocean Engineering, 460 Old Turner St, Blacksburg, VA 24060, AIAA Life Fellow.

${ }^{\ddagger}$ Associate Professor, Kevin T. Crofton Department of Aerospace and Ocean Engineering, 460 Old Turner St, Blacksburg, VA 24060, and AIAA Associate Fellow

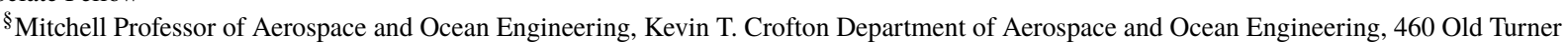
St, Blacksburg, VA 24060, AIAA Fellow. 


$\begin{array}{ll}v_{A-B} & =\text { Average Poisson's ratio of the adhesive and backing of the strain gauge } \\ h_{A} & =\text { Adhesive thickness of the strain gauge } \\ h_{B} & =\text { Backing thickness of the strain gauge } \\ \alpha_{A} & =\text { Thermal expansion coefficient of the adhesive of the strain gauge } \\ \alpha_{B} & =\text { Thermal expansion coefficient of the backing of the strain gauge } \\ \alpha_{S} & =\text { Total corrected strain }\end{array}$

$$
\begin{aligned}
& \text { Subscripts } \\
& \begin{array}{l}
i=\text { Inner } \\
o=\text { Outer } \\
j=\text { Body Number }
\end{array}
\end{aligned}
$$

\section{Introduction}

Sheathed thermocouples (see Fig.1) continue to allow for near-wall temperature sensing of flows involving heat transfer $\checkmark$ and/or chemical reactions. An accurate temperature measurement is key to understanding large energy production within thermal systems such as ramjet and scramjet engines. Robust measurement systems such as thermocouples are needed for flight tests and advanced development applications for their reliability in high-stress environments.

There remains a major need for a quick and accurate way to predict the stresses in the sheath of a metal-clad thermocouple packed with ceramic material at elevated temperatures. An accurate low-order model for predicting the thermal performance of such a thermocouple has already been published [1]. The desire to have high survivability thermocouples at elevated temperatures stems from the frequent failure of these probes during testing. These failures can lead to the loss of time, money, and valuable data. The unpredictability of the structural strength of thermocouples even when using high-fidelity codes at great cost and time limits the ability of the designer to achieve an optimized design for the specific application. High fidelity software may take hours or days to simulate a thermocouple design and produce stress values. To the authors' knowledge, no accurate low-order model has been produced to predict the stresses within the sheath of a thermocouple. The lack of a quick and accurate structural model makes it nearly impossible to quickly design an optimized thermocouple for specific applications. The development of a low-order analytical model with good accuracy that can run in seconds instead of hours or days is an extremely useful tool in the design process in which the goal is an optimized design.

Engineering research practice has often strayed away from an analytical model in favor of a numerical model for their use in capturing 3D effects, especially when involving complicated shapes where common analytical equations break down and are not accurate. In the current case, the mating of a cylindrical body to that of a spherical tip that is filled with potting and wires culminates in complex interactions between the various components that cannot be easily handled by traditional analytical equations.

High-temperature probes cannot use traditional sheath materials as the metal softens to the point of failure or melts altogether. Therefore, precious metals such as platinum, rhodium, and their alloys are used for their high strength and corrosion resistance at elevated temperatures. However, the thermal expansion coefficient of these materials is lower than that of the magnesium oxide which is usually used as the insulator (potting) for the thermocouple. Assuming that the potting has filled the cavity inside the metallic sheath and surrounds the wires, the rapidly expanding potting puts larger and larger pressure onto the inner walls of the slowly expanding sheath as the temperature increases. The large resulting pressure due to the difference in thermal expansion coefficients creates large stresses on the sheath that could eventually lead to yielding and failure.

A)

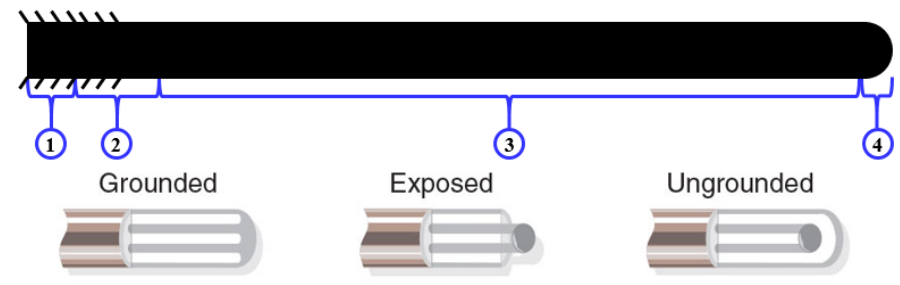

Fig. 1 (A) Thermocouple section classification. (B) Thermocouple junction classifications [2]. 
The metal sheath of a thermocouple can be treated as a thick-walled pressure vessel. Figure 1A shows a thermocouple that has been divided into four distinct sections. Section 1 represents part of the base of the thermocouple that has been clamped into the cooled body securing it in position. Section 2 represents the transition zone between section 1 where the thermocouple is clamped, and section 3 where the thermocouple is no longer clamped, in which effects from sections 1 and 2 are felt. Section 3 is the cylindrical main body of the thermocouple where the largest temperature gradient is located. Finally, section 4 is the nominally spherical tip of a fully enclosed thermocouple.

To solve the stresses in the sheath for all four sections, a linear, low-order model was developed in conjunction with a full, 3D ANSYS mechanical static structural simulation for comparison between the two. A material database was gathered and input into both ANSYS mechanical and the analytical, low-order model. Each material property contains values at various temperatures ranging from room temperature to the highly elevated temperatures both models are run at. It is important to note that both models are limited at elevated temperatures due to the restricted availability of material property data at the highest temperatures of interest. The compiled material database used in this paper can be found in the Appendix in Tables 2-6.

To compare the models, the geometry of the thermocouple must be chosen. More precisely, the junction type must be chosen. The three junction types can be seen in Fig. 1B. A grounded thermocouple has the wires connected to a solid metal tip with no bead. An exposed junction thermocouple has the wires protruding from the tip and the wires are welded together causing a bead to form. The third junction type is the ungrounded thermocouple, where the wires are welded together forming a bead that is then encapsulated inside of the thermocouple below the solid metal tip. In this study, the grounded thermocouple will be used for comparison between the analytical model and a numerical model with one caveat; it is assumed that the connection point between the full metal tip and the cylindrical sheath has a filleted curve as to avoid infinite stress at the junction corners.

\section{Analysis}

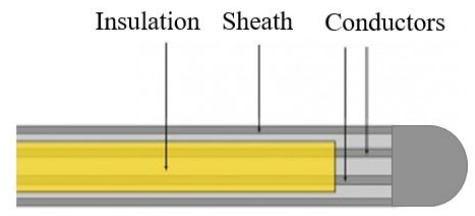

\section{Fig. 2 Thermocouple geometry layout [3].}

A thermocouple consists of two, different material, conductive wires surrounded by an electrically insulating powdered material. Both the wires and insulation are encapsulated within a metallic sheath for protection in harsh environments. In a grounded thermocouple, the wires are attached to the solid spherical tip as seen in Fig. 2. In high-temperature environments, the material combination needed for survivability may mean that the sheath material has a lower thermal expansion coefficient compared to the powdered insulating material. The powdered insulation material is very strong in compression. The expanding powdered insulation pushes on the encapsulating metallic sheath creating a pressure vessel. In this situation, the stresses within the metallic sheath must be known so that the thermocouple doesn't rupture and fail at high temperatures. Predicting these stresses allows for the design of strong sheaths capable of withstanding the harsh outer environment and inner pressure from the expanding insulation material.

Thick-walled, cylindrical and spherical pressure vessel analytical equations can be used as long as the body the equations are applied to is axisymmetric, or spherically symmetric in the case of the tip. A traditional thermocouple is not axisymmetric as the two different wires located inside the sheath are surrounded by potting, and all are different materials. Therefore, simplifying assumptions will be made to make the thermocouple axisymmetric and the tip, spherically symmetric.

The first assumption assumes that the temperature in-plane is constant. The temperature is allowed to vary axially, but at a specific axial location, the temperature cannot vary radially. The second assumption assumes that the material properties only vary with temperature and are constant in-plane. The third assumption invokes a "no-slip" condition applied between the different materials. It is also important to note that if at any point the thermal expansion coefficient of the potting is below that of the sheath, then the low-order approach that is presented in this paper will no longer be valid because the "no-slip" assumption will be invalidated as the sheath pulls away from the potting. Finally, the fourth assumption neglects body forces. 

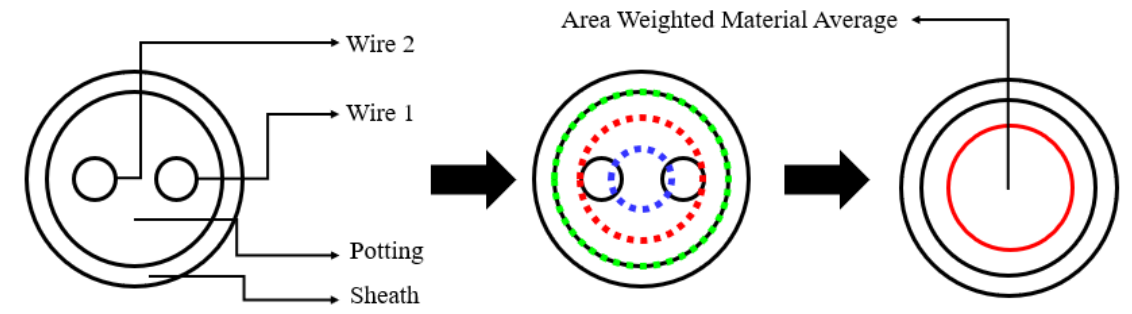

Fig. 3 Thermocouple configuration and area averaging simplification schemes.

Beyond the assumptions above, other simplifications must be made. The left side of Fig. 3 shows the general layout of a thermocouple with the two wires placed inside of the potting surrounded by the sheath. However, as stated, this configuration is not axisymmetric. The first simplification averages the material properties using an area-weighted scheme.

The middle of Fig. 3 shows the different simplification schemes that can be used to make the thermocouple effectively axisymmetric. The blue dotted circle represents scheme one, the red dotted circle represents scheme two, and the green dotted circle represents scheme three. The scheme to make the thermocouple axisymmetric in this simplification moves both wires to the center of the thermocouple and averages their material properties. The second scheme draws a circle around both wires with a radius equal to that of one wire diameter plus half the distance to the second wire. Then both wires plus some of the potting material properties are area-averaged. Finally, the third scheme takes the entire area of the potting and wires and area weight averages the entirety of the area within the sheath. See the three parts of Fig. 3.

To determine which simplification scheme works the best in comparison to the full thermocouple, four, 2D ANSYS mechanical simulations were run. Each was run using a probe with a 0.031-inch diameter with a 0.005 -inch thick sheath and a wire diameter of 0.005 inches. The sheath was made of platinum-rhodium $20 \%$, the potting was magnesium oxide powder, the first wire was platinum-rhodium $10 \%$ and the second wire was platinum-rhodium $30 \%$. These values are representative of common high-temperature thermocouple probes. The entire body was heated to $1832^{\circ} \mathrm{F}\left(1000^{\circ} \mathrm{C}\right)$ which is comparable to a static temperature of approximately $1985^{\circ} \mathrm{F}\left(1085^{\circ} \mathrm{C}\right)$ for a vehicle at an altitude of 30,000 feet going Mach 5. This temperature was limited by available material properties information. The plane stress assumption was used in each run to isolate the effects that the simplification schemes had in the radial and hoop directions only. A full $3 \mathrm{D}$ comparison is made later in this report.

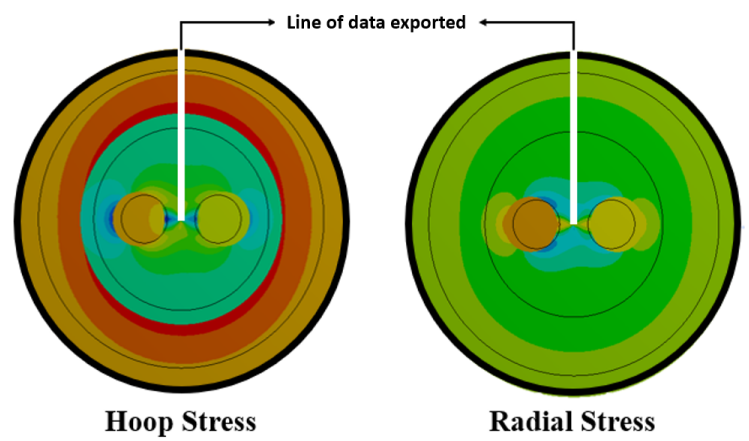

Fig. 4 Simplification study results. Cylindrical coordinates used.

Figure 4 shows the contours produced by ANSYS mechanical for hoop and radial stress. Each contour also includes the non-deformed wire outline for reference. As expected, the inclusion of the wires leads to non-axisymmetric stresses within the sheath. A horizontal line from the center of the thermocouple through a wire and into the sheath will show less stress than if a vertical line (as in Fig. 4) is taken in between the two wires. Since the goal is to predict the highest stress within the sheath, when picking the simplification scheme that works the best, the white vertical line of data will be taken and compared to the different axisymmetric schemes. 
Regardless of what simplification scheme is used, the complex stress gradients seen in Fig. 4 will disappear. Since both wires and the potting are under compression from all sides they are of little concern. Neither the wires nor the potting have stress values that exceed that of their compression strength.

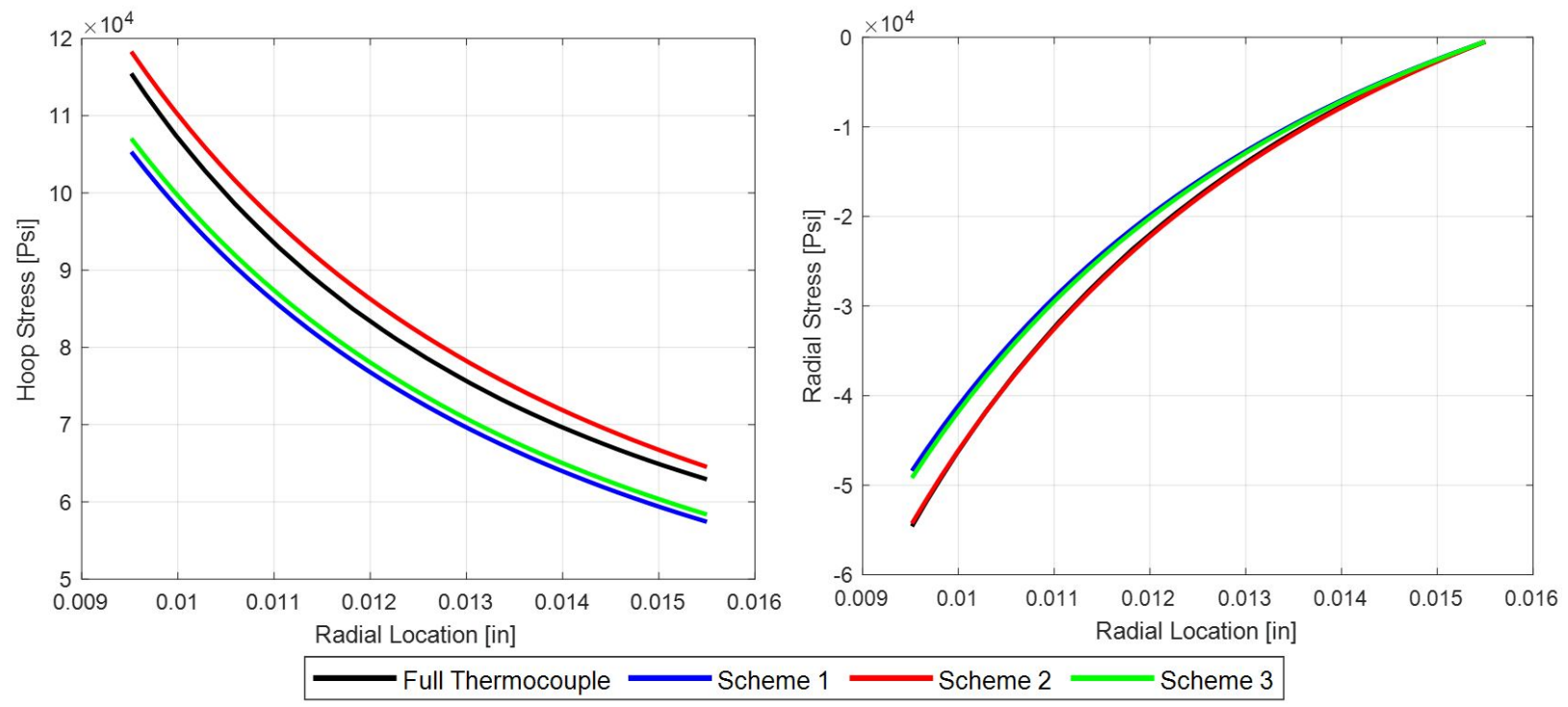

Fig. 5 Simplification study results. Cylindrical coordinates used.

Figure 5 shows on the left-hand side, the hoop stress within the sheath and on the right-hand side, the radial stress within the sheath. Just as in Fig. 3, the black line represents the fully modeled 2D thermocouple, the blue line represents simplification scheme one, the red line, scheme two and the green line, scheme three. It is clear that when comparing hoop and radial stress, scheme two does the best job of being an accurate simplification. This means that modeling a composite rod made up of material properties from both wires and some potting leads to a good approximation of the stresses within the sheath of the fully modeled thermocouple. Now that the thermocouple has been simplified into an axisymmetric body, analytical expressions can be derived to form the basis of an analytical low-order model that predicts the stresses within the sheath of a thermocouple.

\section{A. Main Body (Section 3)}

The main body (section 3 ) is a long cylindrical section made up of the material-averaged, inner rod and the potting ring, all of which are encased inside the metallic sheath. Starting with the 2D equations in the radial and circumferential direction, the first equation corresponds to the radial deformation due to thermal expansion, assuming plane strain [4].

$$
u_{r_{C}}(T, r)_{j}=\alpha_{j}(T) \Delta T r
$$

The next step is to use thick-walled cylindrical pressure vessel equations to account for the radial deformation resulting from the pressure that the inner two materials are placing on the inner surface of the sheath. The thick-walled cylindrical pressure vessel equations being used also assume plane strain and are only valid sufficiently far from the end of the thermocouple [5] leading to a minimum length to diameter ratio requirement of about 10 . This is what gives rise to the transition zone between the base and the main body of the thermocouple. The surrounding ambient pressure is also needed for this calculation. This equation includes Lamé's first and second parameters [6].

$$
u_{r_{C}}(T, r)_{j}=\left(\frac{r}{2\left(\mu_{j}(T)+\lambda_{j}(T)\right)}\right)\left(\frac{\frac{r_{i}^{2}}{r_{o}^{2}} p_{i}(T)-p_{o}(T)}{1-\frac{r_{i}^{2}}{r_{o}^{2}}}\right)+\frac{r_{i}^{2}}{2 \mu_{j}(T) r}\left(\frac{p_{i}(T)-p_{o}(T)}{1-\frac{r_{i}^{2}}{r_{o}^{2}}}\right)
$$

Where,

$$
\lambda_{j}(T)=\frac{E_{j}(T) v_{j}(T)}{\left(1+v_{j}(T)\right)\left(1-2 v_{j}(T)\right)}
$$




$$
\mu_{j}(T)=\frac{E_{j}(T)}{2\left(1+v_{j}(T)\right)}
$$

Equations 1 and 2 account for the 2D effects of the thermally expanding concentric body system. To get the radial deformation due to the axial expansion and make the analytical equations quasi-3D, another equation is needed. Since the "no-slip" condition has been applied at the boundary between each material and the main body section is assumed long, it can be assumed that the axial deformation due to thermal expansion can be found using a form of Eq. 1 again assuming plane strain. Since it is known that the sheath wants to expand less than the potting and the mixed material for the material combinations of interest here, the force needed to keep the "no-slip" condition applied can be found. This leads to three equations that can be simultaneously solved to get the axial stress resulting from the axial thermal expansion.

$$
F_{\text {Sheath }}=F_{\text {Potting }}+F_{\text {Mix }}
$$

Equation 5 indicates that, as expected, the sheath is under tension and the potting plus the mixed material is under compression. Again, this stems from the difference in thermal expansion coefficients.

$$
\begin{gathered}
\alpha_{\text {Sheath }} \Delta T L+\frac{F_{\text {Sheath }} L}{E_{\text {Sheath }} A_{\text {Sheath }}}-\frac{p_{o} L}{E_{\text {Sheath }}}=\alpha_{\text {Potting }} \Delta T L-\frac{F_{\text {Potting }} L}{E_{\text {Potting }} A_{\text {Potting }}}-\frac{p_{o} L}{E_{\text {Potting }}} \\
\alpha_{\text {Potting }} \Delta T L-\frac{F_{\text {Potting }} L}{E_{\text {Potting }} A_{\text {Potting }}}-\frac{p_{o} L}{E_{\text {Potting }}}=\alpha_{\text {Mix }} \Delta T L-\frac{F_{\text {Mix }} L}{E_{\text {Mix }} A_{\text {Mix }}}-\frac{p_{o} L}{E_{\text {Mix }}}
\end{gathered}
$$

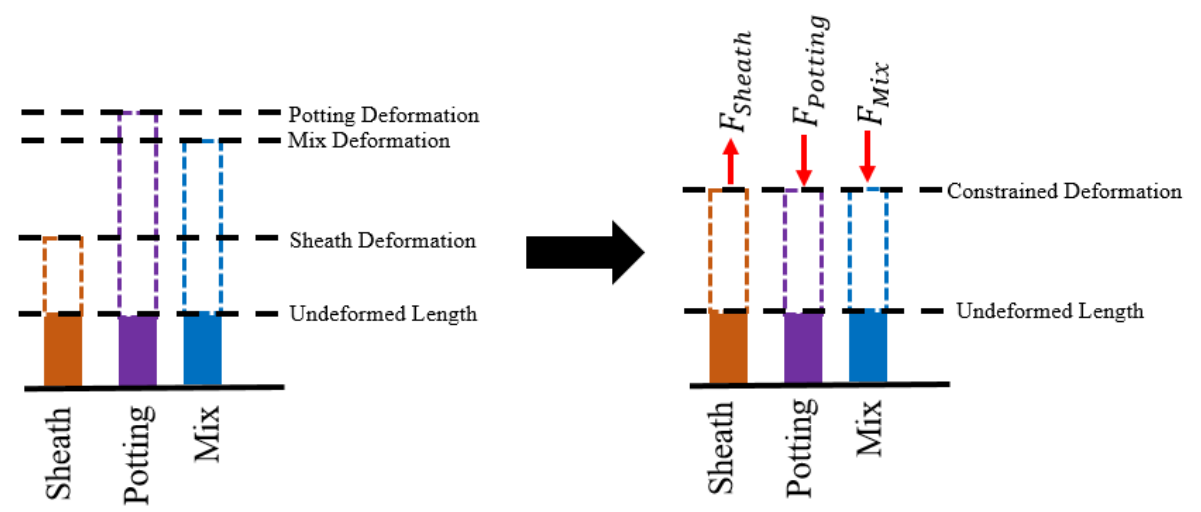

Fig. 6 Application of the No-Slip Condition.

Equations 5 through 7 can be solved simultaneously for $F_{\text {Sheath }}, F_{\text {Potting }}$, and $F_{M i x}$. These forces can then be used to solve for the axial stress resulting from axial thermal expansion.

$$
\sigma_{z z-A x i a l_{j}}= \pm \frac{F_{j}}{A_{j}}-p_{o}
$$

The plus or minus sign in Eq. 8 refers to whether the body being referred to is in tension (+) or compression (-). From here, the radial deformation resulting from the axial expansion can be solved for as seen in Eqs. 9 to 11 .

$$
\sigma_{z z}=E \epsilon_{z z} \rightarrow \frac{\sigma_{z z}}{E}=\epsilon_{z z} \rightarrow \epsilon_{z z}=\frac{\Delta L}{L}
$$

Therefore,

$$
\epsilon_{r r}=-v \epsilon_{z z}=-v\left(\frac{\sigma_{z z}}{E}\right) \rightarrow \epsilon_{r r}=\frac{\Delta r}{r}=-v\left(\frac{\sigma_{z z}}{E}\right)
$$


Finally,

$$
u_{r_{A x i a l}}(T, r)_{j}=-r v_{j}(T)\left(\frac{\sigma_{z z-A x i a l j}(T)}{E_{j}(T)}\right)
$$

Combining the 2D effects (Eqs. 1 and 2) with the radial deformation resulting from the axial expansion (Eq. 11) leads to a quasi $3 \mathrm{D}$ analytical expression for the radial deformation of each body in terms of radial location and temperature in the three-body system (Eq. 12).

$u_{r_{C}}(T, r)_{j}=\alpha_{j}(T) \Delta T r+\left(\frac{r}{2\left(\mu_{j}(T)+\lambda_{j}(T)\right)}\right)\left(\frac{\frac{r_{i}^{2}}{r_{o}^{2}} p_{i}(T)-p_{o}(T)}{1-\frac{r_{i}^{2}}{r_{o}^{2}}}\right)+\frac{r_{i}^{2}}{2 \mu_{j}(T) r}\left(\frac{p_{i}(T)-p_{o}(T)}{1-\frac{r_{i}^{2}}{r_{o}^{2}}}\right)-r v_{j}(T)\left(\frac{\sigma_{z z-A x i a l j}(T)}{E_{j}(T)}\right)$

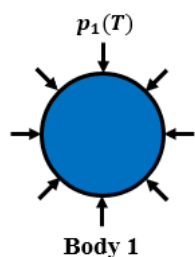

Body 1
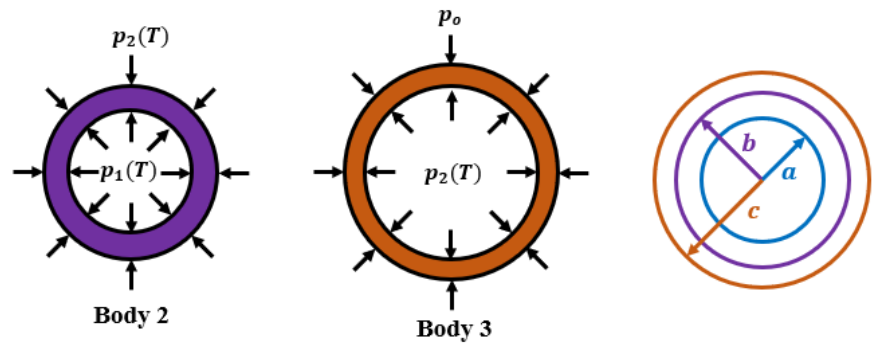

Body 3

Fig. 7 Radii and pressures acting on each body. Not to scale.

To solve for the resulting stresses, the pressures in Eq. 12 must be found. In this three-body system, three pressures are needed: 1) the pressure at the boundary between the inner mixed rod and the potting ring, 2) at the boundary between the potting ring and the sheath ring and 3) finally the outer ambient pressure acting on the sheath ring. Since the outer ambient pressure on the sheath ring is assumed known, only the two inner pressures must be solved, which means two equations resulting from Eq. 12 must be formed.

$$
\begin{aligned}
& u_{r_{C}}(T, a)_{1}=u_{r_{C}}(T, a)_{2} \\
& u_{r_{C}}(T, b)_{2}=u_{r_{C}}(T, b)_{3}
\end{aligned}
$$

Solving Eqs. 13 and 14 simultaneously for $p_{1}(T)$ and $p_{2}(T)$ allows for the unknown stresses to be found using thick-walled cylindrical pressure vessel equations. Both pressures vary with temperature as they are the result of the bodies pushing against each other due to thermal expansion.

$$
\begin{gathered}
\sigma_{r r_{C}}(T, r)_{j}=\frac{\frac{r_{i}^{2}}{r_{o}^{2}} p_{i}(T)-p_{o}(T)}{1-\frac{r_{i}^{2}}{r_{o}^{2}}}-\frac{r_{i}^{2}}{r_{o}^{2}}\left(\frac{p_{i}(T)-p_{o}(T)}{1-\frac{r_{i}^{2}}{r_{o}^{2}}}\right) \\
\sigma_{\theta \theta_{C}}(T, r)_{j}=\frac{\frac{r_{i}^{2}}{r_{o}^{2}} p_{i}(T)-p_{o}(T)}{1-\frac{r_{i}^{2}}{r_{o}^{2}}}+\frac{r_{i}^{2}}{r_{o}^{2}}\left(\frac{p_{i}(T)-p_{o}(T)}{1-\frac{r_{i}^{2}}{r_{o}^{2}}}\right) \\
\sigma_{z z_{C}}(T)_{j}=2 v_{j}(T)\left(\frac{\frac{r_{i}^{2}}{r_{o}^{2}} p_{i}(T)-p_{o}(T)}{1-\frac{r_{i}^{2}}{r_{o}^{2}}}\right) \pm \sigma_{z z-A x i a l}(T)_{j}
\end{gathered}
$$


The radial stress (Eq. 15) and hoop stress (Eq. 16) equations are the cylindrical pressure vessel equations [5]. The axial stress equation uses the cylindrical pressure vessel equation but must also include the axial stress due to the axial thermal expansion. Together, the three stress equations represent the three principal stress directions that can now be analytically solved. Each equation will use $p_{i}(T)$ which is the inner pressure acting on that body, $j$, and $p_{o}(T)$ which is the outer pressure acting on that body. As can be seen in Fig. 7, body 1 has no inner pressure, since it is a solid body. Consequently, it also has no inner radius, $r_{i}$. Body 3 has both inner and outer pressure, however, the outer pressure does not need to be solved as it is an input based on ambient conditions.

The radial stress and hoop stress for body 1 simplify down to just $-p_{1}(T)$ since it is a solid body. This means that for body 1, radial, hoop and axial stress do not vary radially, only axially. The axial variation comes into effect with an axial temperature gradient. If no axial temperature gradient is present the stresses will be constant through body 1 . On the other hand, the radial and hoop stress in bodies 2 and 3 vary radially as both bodies have inner and outer pressure as well as an inner and outer radius. However, axial stress remains constant in the radial direction. Again, all three stresses will vary axially if an axial temperature gradient is present, but axial stress will never vary radially based on the assumptions made.

\section{B. Clamped Base (Section 1)}

The clamped base section of a thermocouple uses the same equations as the main body of the thermocouple. However, the bodies that the thermocouple is attached to must now be taken into account. A thermocouple with a shield around it is generally clamped into the base of a shield and then the shield is welded to another structure as seen in Fig. 8. The shield base and mounting structure geometry and material properties must be taken into account to get accurate stress calculations within the thermocouple sheath in this section.

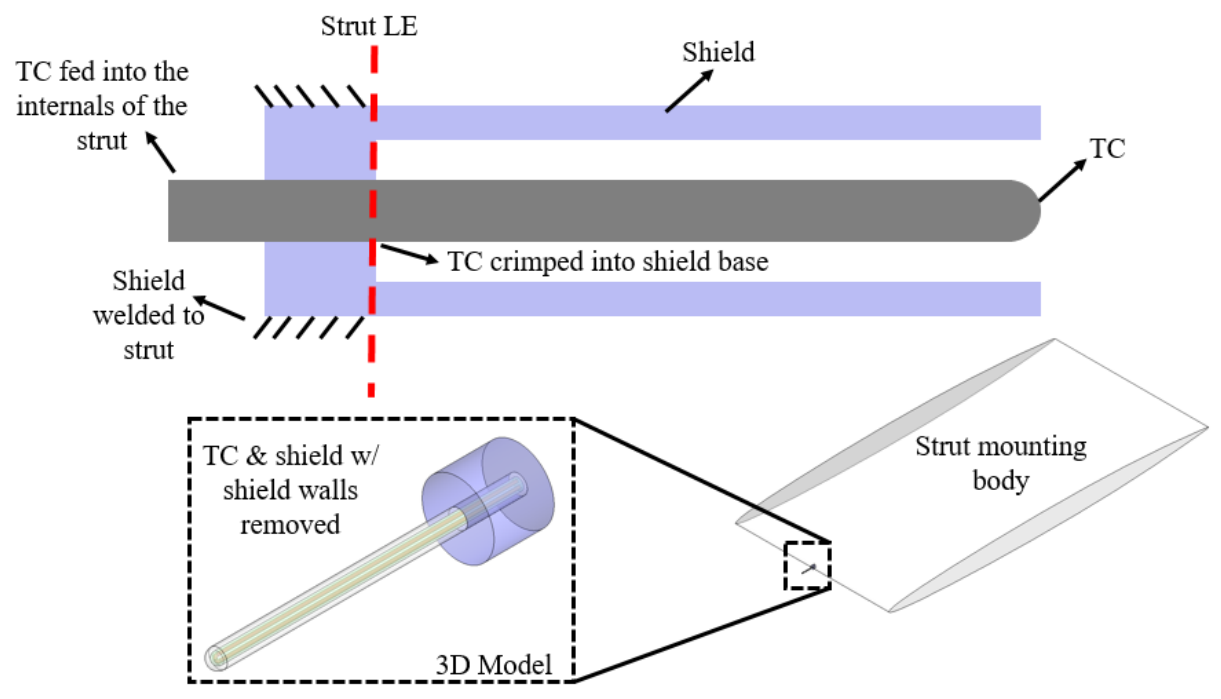

Fig. 8 Thermocouple base geometry and mounting structure.

As stated in the above section, the analytical equations for the desired low-order model require the bodies that are being modeled to be axisymmetric. The shield base is already axisymmetric, however, the strut mounting body is not. The strut is generally a very large structure compared to the thermocouple, therefore most of it does not need to be modeled to get an accurate result. To trim the strut size down, a cut at the end of the shield base is made and then another cut is made to make a small square piece containing the leading edge as well as the entire thermocouple and shield base.

The second simplification that must be made is taking the trimmed strut and converting it into an axisymmetric shape. To use the analytical equations shown in the previous section, the strut must be simplified down into a cylinder. The square cross-sectional area of the remaining strut, thermocouple combination can be found. The square cross-sectional area can then be equated to a circular cross-sectional area. From this, a cylinder can be extruded the length of the shield base to form the simplified strut body as seen on the right-hand side of Fig.9. The result is an axisymmetric five body system as seen in Fig. 9 and Fig. 10. 


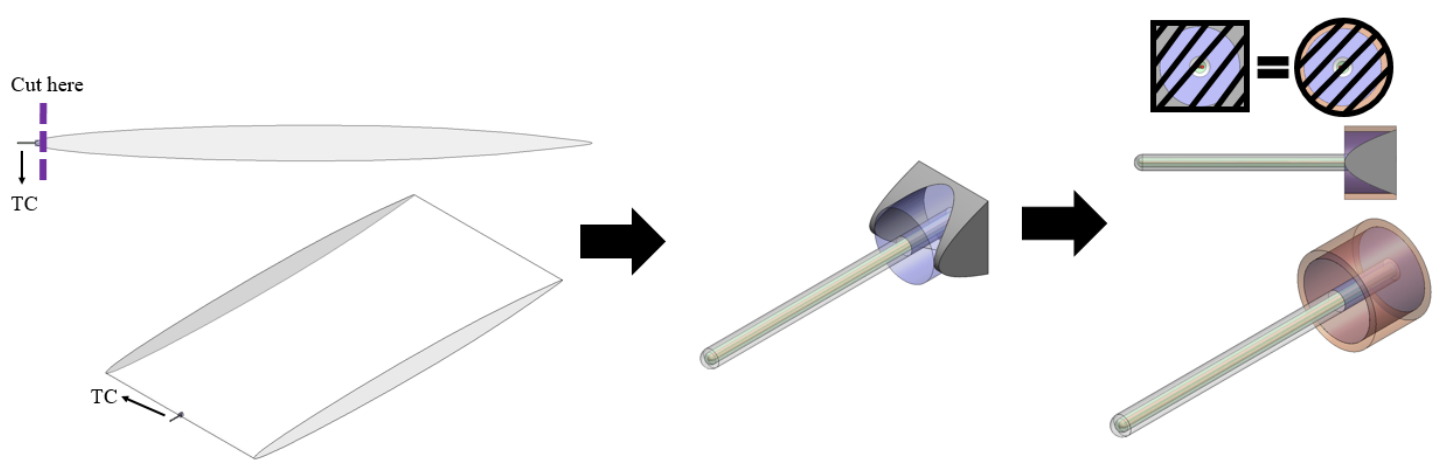

Fig. 9 Base simplification technique.
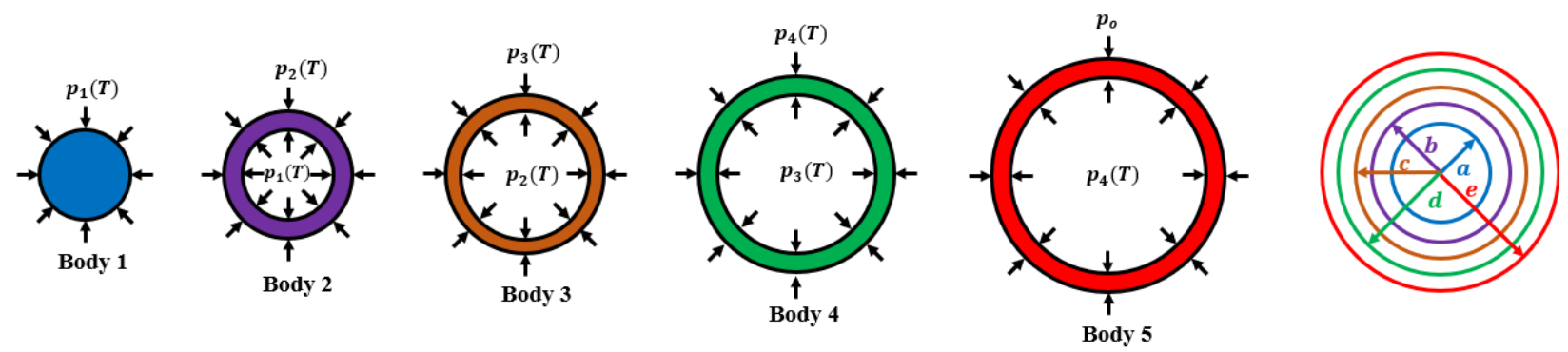

Fig. 10 Five body system with four unknown pressures. Not to scale.

To use Eqs. 15 through 17 to find the stresses in each body, the four unknown pressures must be found. Using Eq. 12 at each boundary between the bodies will give four equations to solve for the four unknown pressures.

$$
\begin{aligned}
& u_{r_{C}}(T, a)_{1}=u_{r_{C}}(T, a)_{2} \\
& u_{r_{C}}(T, b)_{2}=u_{r_{C}}(T, b)_{3} \\
& u_{r_{C}}(T, c)_{3}=u_{r_{C}}(T, c)_{4} \\
& u_{r_{C}}(T, d)_{4}=u_{r_{C}}(T, d)_{5}
\end{aligned}
$$

Now that the four unknown pressures have been solved for, Eqs. 15 through 17 can be used to find the stress values at every location within the clamped base. However, the plane strain assumption will not be satisfied for the axial stress because of how short the base is compared to how wide the mounting strut body is. In this case, it is expected that the axial stress in the base incurs higher error in comparison with the radial and hoop stress predictions. This will be explored further in the results section.

\section{Transition Zone (Section 2)}

The transition zone is the zone between the base of the thermocouple that is clamped into the shield base and the main body of the thermocouple. No analytical equation by itself can capture the effects of both the clamped base and the main body. Therefore, a linear averaging technique based on axial location is used to get an approximate result.

The transition zone starts approximately halfway up the shield base and continues for approximately the same distance into the main body of the thermocouple. Looking at Fig. 11, to the left of the green line, only the clamped base equations are used, to the right of the blue line, only the main body equations are used. In between the two lines, the results from each equation are linearly averaged using location weighting. Therefore at the green line, only the clamped 
base equations will be used, and at the blue line, only the main body equations will be used. The distance between the two points will be referred to as $L_{\text {Trans }}$ measured from the green line to the blue line. The distance between the green line and the plane being solved for will be referred to as $Z_{L o c}$ which has a minimum value of 0 and a maximum value of $L_{\text {Trans. }}$. The resulting combined analytical equations to be used within this transition zone can be seen below.

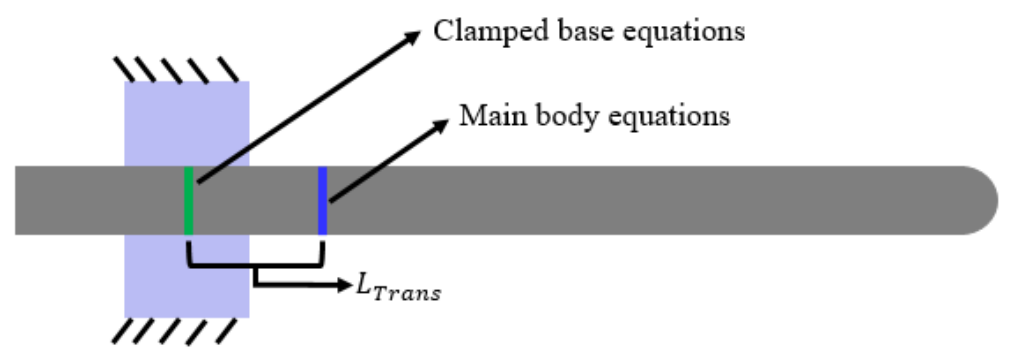

Fig. 11 The transition zone occurs between the green and blue lines.

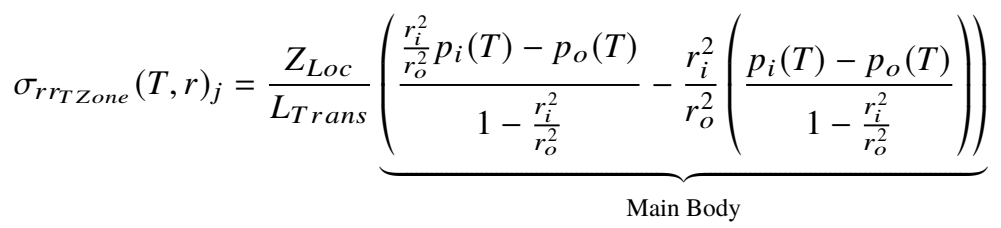

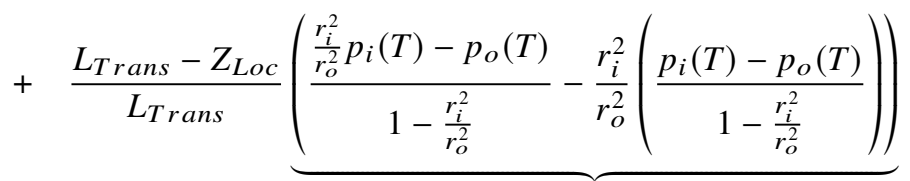

$$
\begin{aligned}
& \text { Clamped Base }
\end{aligned}
$$

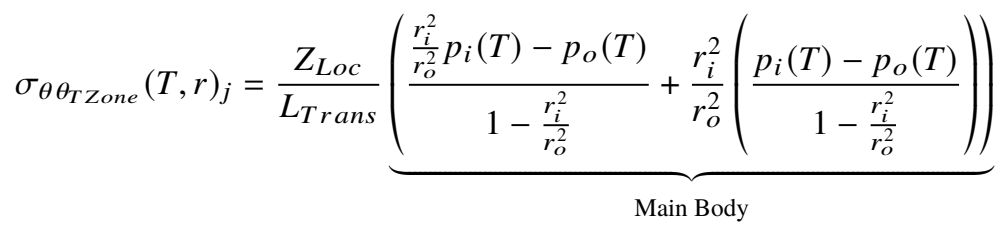

$$
\begin{aligned}
& +\frac{L_{\text {Trans }}-Z_{\text {Loc }}}{L_{\text {Trans }}} \underbrace{\left(\frac{r_{i}^{2}}{r_{o}^{2}} p_{i}(T)-p_{o}(T)\right.}_{\text {Clamped Base }} \underbrace{\left.r_{i}^{2}\left(\frac{p_{i}(T)-p_{o}(T)}{r_{o}^{2}}\right)\right)}_{1-\frac{r_{i}^{2}}{r_{o}^{2}}} \\
& \sigma_{\theta \theta_{\text {TZone }}}(T, r)_{j}=\frac{Z_{\text {Loc }}}{L_{\text {Trans }}} \underbrace{\left(2 v_{j}(T)\left(\frac{\frac{r_{i}^{2}}{r_{o}^{2}} p_{i}(T)-p_{o}(T)}{1-\frac{r_{i}^{2}}{r_{o}^{2}}}\right) \pm \sigma_{z z-\text { Axial }}(T)_{j}\right)}_{\text {Main Body }} \\
& +\frac{L_{\text {Trans }}-Z_{L o c}}{L_{\text {Trans }}} \underbrace{\left(2 v_{j}(T)\left(\frac{\frac{r_{i}^{2}}{r_{o}^{2}} p_{i}(T)-p_{o}(T)}{1-\frac{r_{i}^{2}}{r_{o}^{2}}}\right) \pm \sigma_{z z-A x i a l}(T)_{j}\right)}_{\text {Clamped Base }}
\end{aligned}
$$




\section{Tip (Section 4)}

Unlike the other three sections that are concentric cylindrical bodies, the tip section is a spherical concentric body system. Therefore, the equations previously presented will no longer apply. Thick-walled spherical pressure vessel equations alone will not work either. This is because the spherical equations assume the body to be spherically symmetric. In this case, a dome has been set on top of and connected to, a long cylindrical body that is not spherically symmetric. To accurately capture the stresses within the tip, the effects from the cylindrical section of the thermocouple must be accounted for. To do this, both the main body cylindrical stress and the tip spherical stress must be combined.

The spherical pressure vessel equations already account for all of the 3D effects felt in the tip. This means there is no need to do a quasi, 3D addition to the radial deformation equation like what is done to the cylindrical pressure vessel equations. Looking at the spherical pressure vessel equations [5], they are very similar to those used in the main body and clamped base sections. Again, an axisymmetric concentric body system must be used to solve for the unknown pressures as a result of the bodies pressing against each other due to thermal expansion. Starting with the radial expansion equation, the radial deformation due to thermal expansion, Eq. 1, has been added to that of the radial deformation due to internal and external pressures resulting from the thermal expansion mismatch.

$$
u_{r_{S}}(T, r)_{j}=\alpha_{j}(T) \Delta T r+\left(\frac{r}{2 \mu_{j}(T)+3 \lambda_{j}(T)}\right)\left(\frac{\frac{r_{i}^{3}}{r_{o}^{3}} p_{i}(T)-p_{o}(T)}{1-\frac{r_{i}^{3}}{r_{o}^{3}}}\right)+\frac{r_{i}^{3} r_{o}^{3}}{4 \mu_{j}(T) r^{2}}\left(\frac{p_{i}(t)-p_{o}(T)}{r_{o}^{3}-r_{i}^{3}}\right)
$$

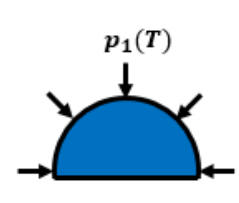

Body 1

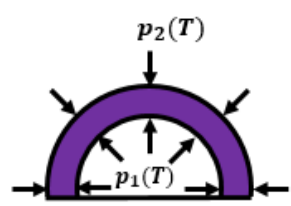

Body 2

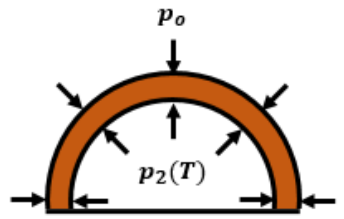

Body 3

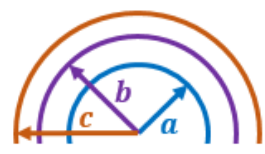

Fig. 12 The three-body system with two unknown pressures. Not to scale.

Just like the previous procedures, Eq. 25 can be used at the boundaries of each body to solve for the two unknown pressures, $p_{1}(T)$ and $p_{2}(T)$ shown in Fig. 12. Doing this will result in two equations to be simultaneously solved.

$$
\begin{gathered}
u_{r_{S}}(T, a)_{1}=u_{r_{S}}(T, a)_{2} \\
u_{r_{S}}(T, b)_{2}=u_{r_{S}}(T, b)_{3} \\
\sigma_{r r_{S}}(T, r)_{j}=\frac{r_{i}^{3} p_{i}(T)-r_{o}^{3} p_{o}(T)}{r_{o}^{3}-r_{i}^{3}}-\frac{r_{i}^{3} r_{o}^{3}}{r^{3}}\left(\frac{p_{i}(T)-p_{o}(T)}{r_{o}^{3}-r_{i}^{3}}\right) \\
\sigma_{\theta \theta_{S}}(T, r)_{j}=\sigma_{\phi \phi_{S}}(T, r)_{j}=\frac{r_{i}^{3} p_{i}(T)-r_{o}^{3} p_{o}(T)}{r_{o}^{3}-r_{i}^{3}}+\frac{r_{i}^{3} r_{o}^{3}}{2 r^{3}}\left(\frac{p_{i}(T)-p_{o}(T)}{r_{o}^{3}-r_{i}^{3}}\right)
\end{gathered}
$$

Having solved for the pressures, Eqs. 28 and 29 [5] are used to solve for the stress in the tip as if it is spherically symmetric. Assuming spherically symmetric means that both the theta and phi directions have the same stress. However, as stated previously this does not accurately reflect the stress in the tip of a thermocouple due to the connection of the main body of the thermocouple. To capture the effects of the cylindrical section on the spherical section, a special case will be taken advantage of.

To combine the thick-walled spherical pressure vessel equations and the thick-walled cylindrical pressure vessel equations, a special property of the spherical coordinate system will be taken advantage of. If point $K$ in Fig. 13 is placed on the z-axis, then the spherical pressure vessel equations can be transformed into cylindrical pressure vessel equations. Placing the point $K$ on the z-axis in both the spherical coordinate system and the cylindrical coordinate system makes the angle theta zero. In the spherical coordinate system, the angle phi is zero, and in the cylindrical 
coordinate system, the length $r$ becomes zero. This leaves the $r$ length in the spherical coordinate system pointing straight up, in line with the z-axis. This means that the $r$ axis in the spherical coordinate system is now the same thing as the $z$ length in the cylindrical coordinate system.

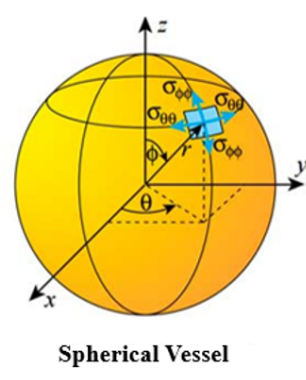

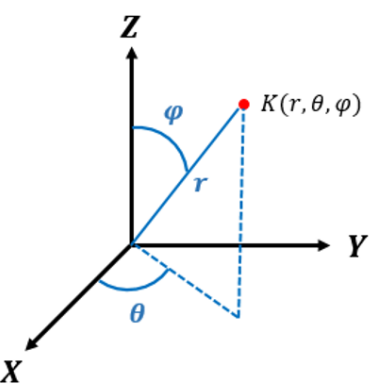

Spherical Coordinates

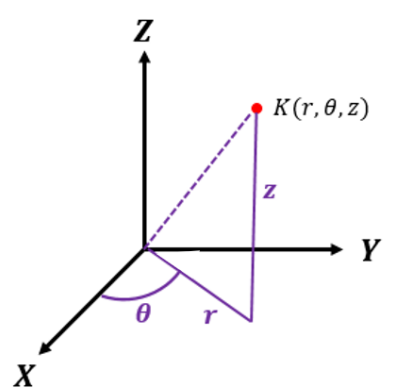

Cylindrical Coordinates

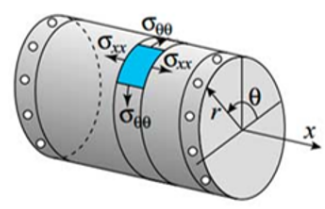

Cylindrical Vessel

Fig. 13 Spherical and cylindrical axis comparison [7].

In fewer words, by using the spherical pressure vessel equations just along the z-axis, the resulting stresses can be transformed into cylindrical coordinate stresses. Theta in spherical coordinates is equal to theta in cylindrical coordinates. $r$ in spherical coordinates is equal to $z$ in cylindrical coordinates. Finally, phi in spherical coordinates is equal to $r$ in cylindrical coordinates.

By making use of the special properties of the spherical pressure vessel equations along the z-axis, the spherical pressure vessel equations and the cylindrical pressure vessel equation can be combined. Looking at Fig. 14, the red line is the line on which the spherical pressure vessel equations are used to calculate the stresses at every radial location. The blue line is the line on which the cylindrical pressure vessel equations are used to calculate the stresses at every radial location. Since both sets of equations can be thought of as in the cylindrical coordinate system, the stresses can be averaged together to get an approximate solution of the main body effects on the tip. Every angular location uses a linear angle weighted average.

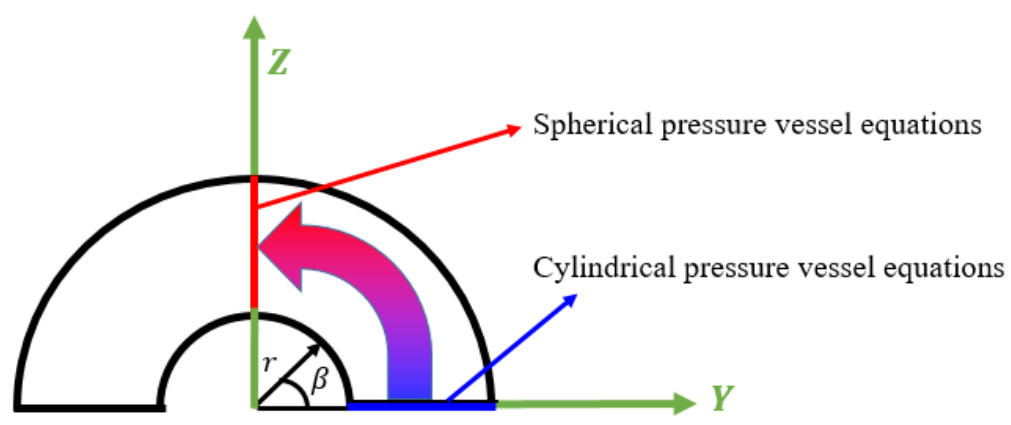

Fig. 14 Tip equations using angle weighted averaging.

The resulting stresses in the tip of the thermocouple, in cylindrical coordinates, end up being a linear angle averaged sum using both the spherical pressure vessel equations (now in cylindrical coordinates) and the cylindrical pressure vessel equations. The resulting averaged equations can be seen below. 


$$
\begin{aligned}
\sigma_{r r_{T i p}}(T, r)_{j} & =\frac{\beta}{\pi / 2} \underbrace{\left(\frac{r_{i}^{3} p_{i}(T)-r_{o}^{3} p_{o}(T)}{r_{o}^{3}-r_{i}^{3}}-\frac{r_{i}^{3} r_{o}^{3}}{r^{3}}\left(\frac{p_{i}(T)-p_{o}(T)}{r_{o}^{3}-r_{i}^{3}}\right)\right)}_{\text {Spherical Pressure Vessel }} \\
+ & \frac{(\pi / 2)-\beta}{\pi / 2} \underbrace{\left(\frac{\frac{r_{i}^{2}}{r_{o}^{2}} p_{i}(T)-p_{o}(T)}{1-\frac{r_{i}^{2}}{r_{o}^{2}}}-\frac{r_{i}^{2}}{r_{o}^{2}}\left(\frac{p_{i}(T)-p_{o}(T)}{1-\frac{r_{i}^{2}}{r_{o}^{2}}}\right)\right)}_{\text {Cylindrical Pressure Vessel }}
\end{aligned}
$$

$$
\begin{aligned}
\sigma_{\theta \theta_{T i p}}(T, r)_{j} & =\frac{\beta}{\pi / 2} \underbrace{\left(\frac{r_{i}^{3} p_{i}(T)-r_{o}^{3} p_{o}(T)}{r_{o}^{3}-r_{i}^{3}}+\frac{r_{i}^{3} r_{o}^{3}}{2 r^{3}}\left(\frac{p_{i}(T)-p_{o}(T)}{r_{o}^{3}-r_{i}^{3}}\right)\right)}_{\text {Spherical Pressure Vessel }} \\
+ & \frac{(\pi / 2)-\beta}{\pi / 2} \underbrace{\left(\frac{\frac{r_{i}^{2}}{r_{o}^{2}} p_{i}(T)-p_{o}(T)}{1-\frac{r_{i}^{2}}{r_{o}^{2}}}+\frac{r_{i}^{2}}{r_{o}^{2}}\left(\frac{p_{i}(T)-p_{o}(T)}{1-\frac{r_{i}^{2}}{r_{o}^{2}}}\right)\right)}_{\text {Cylindrical Pressure Vessel }}
\end{aligned}
$$

$$
\begin{array}{r}
\sigma_{z z_{T i p}}(T, r)_{j}=\frac{\beta}{\pi / 2} \underbrace{\left(\frac{r_{i}^{3} p_{i}(T)-r_{o}^{3} p_{o}(T)}{r_{o}^{3}-r_{i}^{3}}+\frac{r_{i}^{3} r_{o}^{3}}{2 r^{3}}\left(\frac{p_{i}(T)-p_{o}(T)}{r_{o}^{3}-r_{i}^{3}}\right)\right)}_{\text {Spherical Pressure Vessel }} \\
+\frac{(\pi / 2)-\beta}{\pi / 2} \underbrace{\left(2 v_{j}(T)\left(\frac{\frac{r_{i}^{2}}{r_{o}^{2}} p_{i}(T)-p_{o}(T)}{\left.1-\frac{r_{i}^{2}}{r_{o}^{2}}\right)}\right) \pm \sigma_{z z-\text { Axial }}(T)_{j}\right)}_{\text {Cylindrical Pressure Vessel }}
\end{array}
$$

\section{Comparison of Analytical and Numerical Results}

High-fidelity static, structural simulations have been run in ANSYS mechanical to compare a full-fledged numerical method with the analytical methods described in the previous section for a representative probe design. In both cases, the thermocouple is placed into the shield base which is then attached to a symmetric biconvex airfoil with a slightly curved leading edge with a thickness of 6\%, a chord of 12 inches and a span of 6 inches (Fig. 15). The airfoil profile can be found in Table 7. The shield base has a length of 0.1 inches and a diameter of 0.12 inches. The thermocouple has a diameter of 0.031 inches and has a sheath thickness of 0.005 inches with wire diameters of 0.005 inches with a 0.005 -inch spacing in between the two wires. The thermocouple has a length to diameter ratio of 15 giving it a total length past the shield base of 0.465 inches. The total length of the thermocouple is 0.565 inches including the clamped base section. The sheath is made out of platinum-rhodium $20 \%$ whereas the two wires are made of platinum-rhodium $10 \%$ and platinum-rhodium 30\% respectively. Magnesium oxide is used for the potting material. The biconvex strut is made out of Inconel 600, and the shield base is made out of platinum-rhodium 20\%, the same as the sheath.

The materials chosen make for a special combination in that platinum-rhodium is strong at high temperatures and corrosion resistant, however, it has a relatively low thermal expansion coefficient. Its thermal expansion coefficient is lower than that of the magnesium oxide powder over the temperature ranges where data is available. Thus, the resulting system is exactly what the analytical equations presented in the last section can be applied to. Assuming that the magnesium oxide powder is fully packed into the thermocouple, it satisfies the "no-gap" condition required. By clamping the thermocouple into the base and assuming the potting is fully packed into the sheath, the potting cannot move and thus satisfies the "no-slip" condition. 


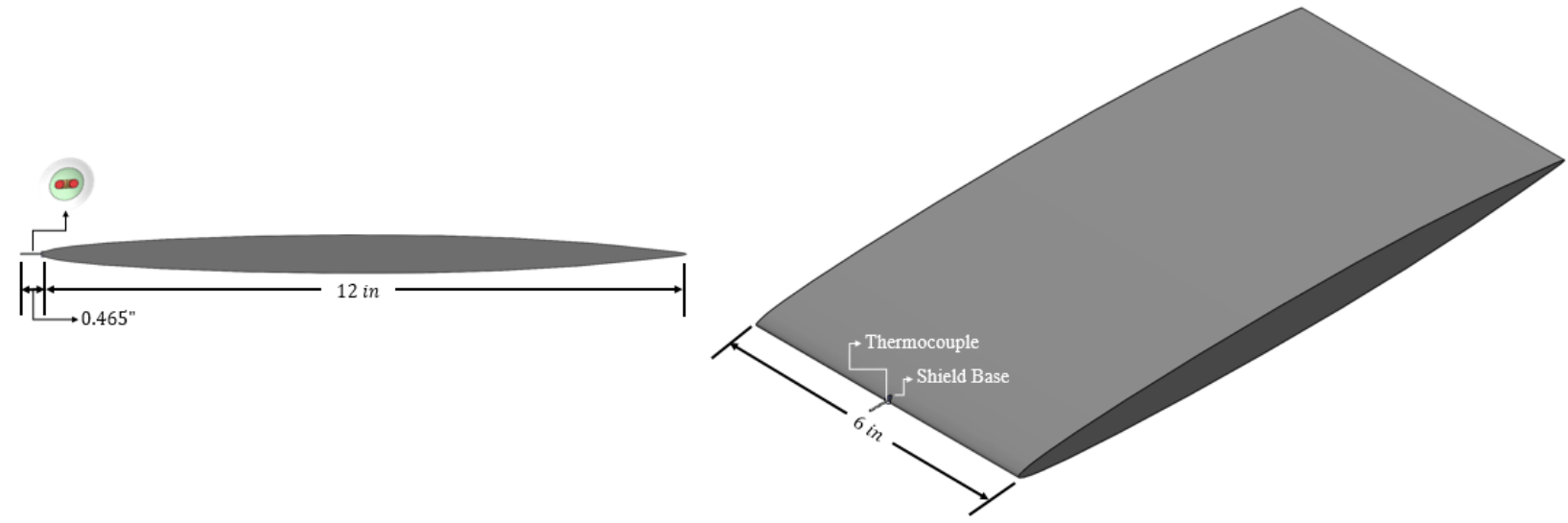

Fig. 15 3D Model in ANSYS mechanical.

ANSYS mechanical allows for a high-fidelity 3D model to be directly imported. The model was made in SolidWorks, converted into a Parasolid file and then imported into ANSYS. Within ANSYS the material property database tables are input and then each part is assigned a material. ANSYS mechanical allows for the material properties to vary with temperature and uses linear interpolation between data points to acquire the properties at specific temperatures. A full, high-fidelity 3D model is used to validate all assumptions made within the low-order model. An arbitrary hypersonic airfoil geometry is chosen such as the biconvex airfoil previously mentioned, to show that the low-order model simplifying geometry assumptions will work for any airfoil type shape.

The analytical low-order model cannot take in a SolidWorks file like ANSYS, rather, user input is needed for computation. Just like in ANSYS, the material property database tables had to be input into the code to assign parts specific materials. The low-order model also uses linear interpolation between data points to get values at specific temperatures. Unlike the high-fidelity model that treats the thermocouple wires as separate entities, the low-order model must use the inner mix simplifying technique. The low-order model computes the area-averaged material properties based on the user inputs of the wire diameter, wire materials, and the potting material. The resulting model that the low-order model must solve looks like Fig. 16.

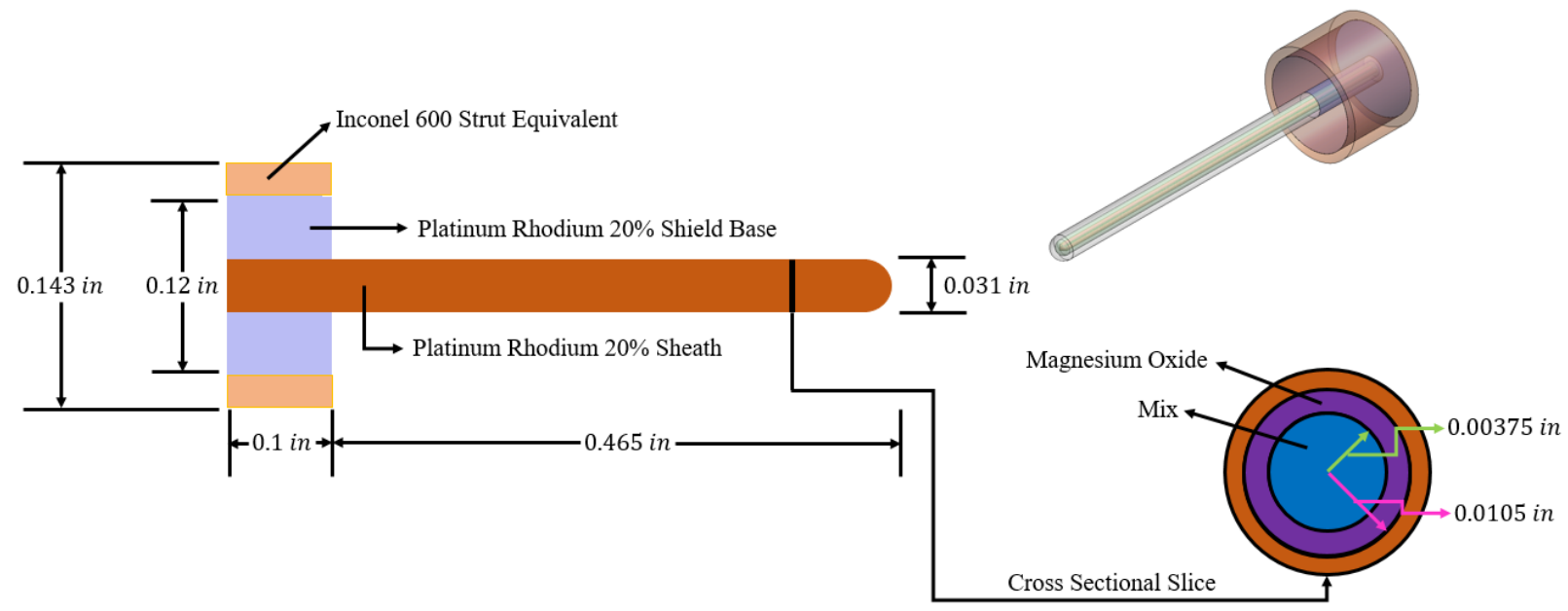

Fig. 16 The equivalent model used with the analytical equations in the low-order model.

Since a grounded probe is being modeled, no material averaging is needed in the solid metallic tip. The clamped base has one solid rod and four rings made up of the inner mix, the potting, the sheath, the shield base and finally the strut equivalent ring. It is important to note that because the sheath and the shield base are made of the same material 
they can be combined to simplify the problem into one solid rod and three rings (four bodies). The main body has one solid rod and two rings made up of the inner mix, the potting and the sheath. Finally, the tip is made up of one solid inner dome with two outer hollowed domes surrounding it. Even though the tip is one material it is important to keep the body count in the tip the same as the main body to accurately capture the effects from the main body sections three individual parts on the tip. Overall, the low-order model will be used to solve ten bodies with analytical equations and then compared to the high-fidelity results produced by ANSYS mechanical.

\section{A. ANSYS Mechanical Modeling and Statistics}

ANSYS mechanical is a numerical finite element analysis solver used in structural analysis and design. As stated previously, ANSYS mechanical allows for the user to import Parasolid objects from SolidWorks for use in the analysis. After the model is imported, each part is assigned a material that pulls from a user-entered material database. The material database has material properties at various temperatures where the properties are linearly interpolated for use in the calculations. This is important because it allows for the same materials database to be used in both the full simulation and with the low-order analytical model. Contact boundary conditions must be set up between the different parts. In this case, six of these conditions are imposed on the model. Bonded boundary conditions between the shield base and strut, the sheath and shield base, the potting to the sheath, both wires to the tip and finally the wires to the potting are used.

Table 1 ANSYS mechanical meshing statistics.

\begin{tabular}{|c|c|}
\hline Mesh Region & Element Size (in) \\
\hline Strut & $7.874 \mathrm{E}-2$ \\
Shield Base & $2.9528 \mathrm{E}-3$ \\
Thermocouple & $1.9685 \mathrm{E}-3$ \\
Shield Base/Strut Contact & $7.874 \mathrm{E}-4$ \\
Sheath/Shield Base Contact & $7.874 \mathrm{E}-4$ \\
Potting/Sheath Contact & $7.874 \mathrm{E}-4$ \\
Wire/Wire Contact & $7.874 \mathrm{E}-4$ \\
Wires/Tip Contact & $7.874 \mathrm{E}-4$ \\
Wires/Potting Contact & $7.874 \mathrm{E}-4$ \\
\hline \hline Total Elements & $\mathbf{6 , 8 0 7 , 9 7 9}$ \\
Total Nodes & $\mathbf{1 8 , 3 8 3 , 9 3 6}$ \\
\hline
\end{tabular}

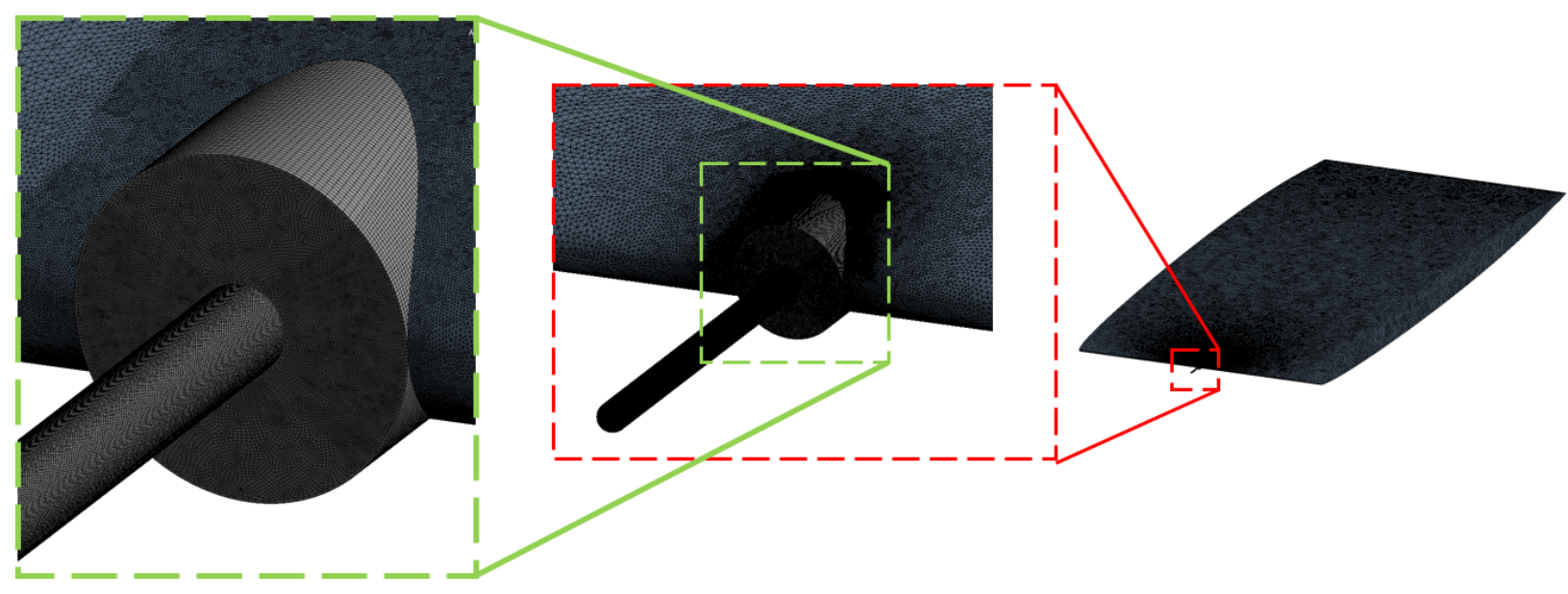

Fig. 17 3D Meshing in ANSYS mechanical. 
The next and most important step is to mesh the model. As the strut is a very large body compared to the thermocouple, the mesh used there is coarse compared to that which is used for the thermocouple and its components. However, each contact boundary is given a refined mesh so that the interaction between each body can be accurately captured. This means that the mesh size of the strut around the shield base is also refined. Ultimately, almost 7 million tetrahedral elements and approximately 18 million nodes are used to mesh the entire 3D model (Table 1 and Fig. 17).

Finally, the displacement boundary condition must be imposed on the body to anchor it in space and prevent rigid body motion. To let the model expand freely in every direction only one displacement boundary condition is used to anchor the model as shown in Fig. 18. A stationary point that cannot be moved or twisted is used on the bottom of the thermocouple in between the two wires. Using this displacement boundary condition along with the bonded contact regions allowed the model to freely expand in every direction accurately capturing the effects of the thermally expanding bodies. As stated previously, the thermocouple is clamped into the shield base and then the shield base is welded to the strut. This configuration will expand together due to the material properties of the system.

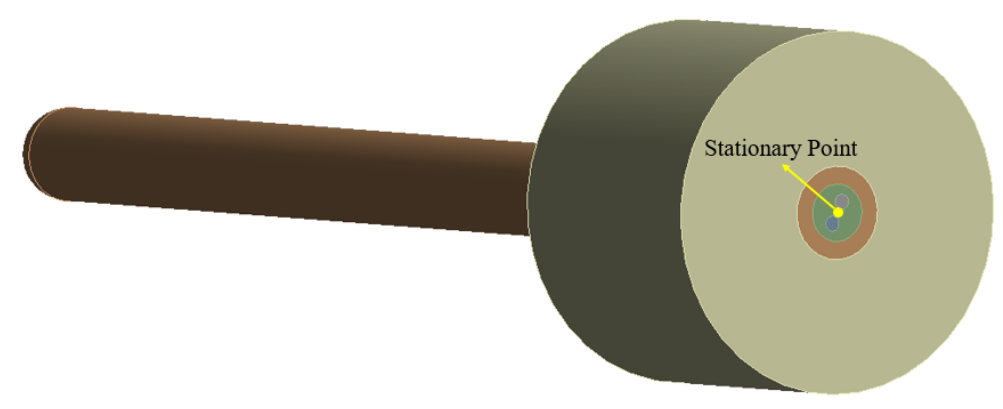

Fig. 18 Displacement boundary condition.

\section{B. High-Fidelity Simulation and Low-Order Model Results Comparison}

In high-temperature applications, cooled thermocouples are used to keep the sensor's structural strength. A cooled thermocouple is one in which its base is cooled to a temperature that is much lower than that of the surrounding air. The overall effect on the thermocouple is the introduction of a large temperature gradient in the axial direction. Methods for calculating the thermal performance of such a probe can be found in Ref. [1].

A realistic way to relate ANSYS mechanical and the low-order model to the physical world is to impart an axial temperature gradient along the thermocouple starting at a low temperature where the thermocouple comes out of the cooled shield base and ending at a much higher temperature at the tip of the thermocouple. A linear temperature gradient is adopted instead of a combined fluid-structure analysis to find a more physically accurate temperature gradient for its simplicity and ease of implementation into the low-order model. The entire shield base and strut are held at the same temperature as the base of the thermocouple that has just exited the shield base (lowest temperature). The tip of the thermocouple is kept at $2000^{\circ} \mathrm{F}\left(1093^{\circ} \mathrm{C}\right)$ while the base is kept at $1500^{\circ} \mathrm{F}\left(816^{\circ} \mathrm{C}\right)$. These temperatures were chosen as they represent typical static temperature conditions at approximately 30,000 feet flying at Mach 5. Note that some of the materials used did not have material property values at these temperatures. In that case, the last known value was used. It should be said that since no fluid-structure interaction simulations were run, the ambient pressure is assumed to be the same as the reference pressure of one atmosphere. This resulted in an outer gauge pressure of zero in the structural model. An ambient gauge pressure of zero means that the radial stress on the outer surface of the sheath in both models is expected to be zero in the main body and tip regions. The ANSYS results were imported into MATLAB for post-processing and comparison with the low-order model.

An important characteristic of the low-order analytical model is that it assumes everything is axisymmetric. It cannot deal with complex geometry like the potting in between both thermocouple wires. Figure 19 is a cross-sectional slice showing stress contours of the complex geometry ANSYS simulation on the left and an ANSYS simplified model simulation on the right. By eliminating the complex wire/potting geometry and creating an area-weighted material averaged inner rod, the model becomes axisymmetric as expected. The sheath stresses in both models remain very close to each other even with the simplification used in the low-order model that was applied in ANSYS. This is what allows the low-order model to be accurate in predicting the stresses within the sheath. The simplified ANSYS simulation was run as well as the high-fidelity simulation for direct comparison with the low-order model results. 


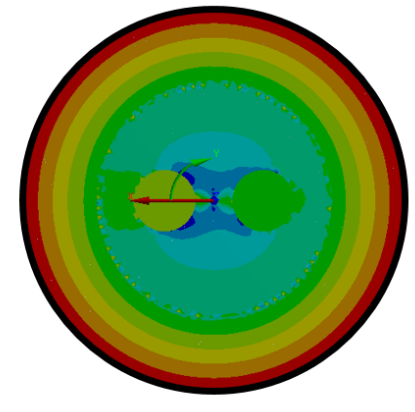

ANSYS High Fidelity Model

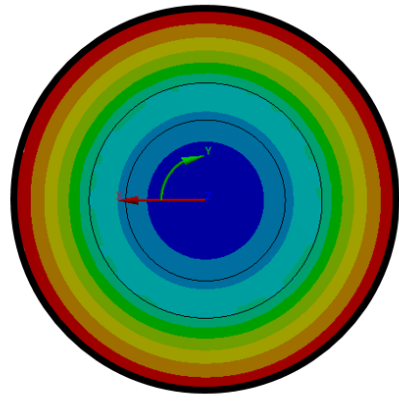

ANSYS Simplified Model

Fig. 19 High-fidelity comparison with the simplified model in ANSYS. Showing hoop stress.

Figure 20 shows a 2D cross-sectional slice from the main body of the thermocouple using stress results from the ANSYS simulations with the high-fidelity model result in blue and the simplified model results in black. Additionally, the red line signifies the result produced by the low-order model. As expected, the simplified geometry ANSYS model and the low-order model line up almost exactly at every radial location including the potting and mix regions, however the high-fidelity ANSYS model results do not match except within the sheath. The wire and potting stresses are of little concern as these components are always under compression and will not fail under this kind of load. That is why the sheath stress values are the main concern. The maximum percent error in the radial stress and hoop stress for this cross-sectional cut is approximately $2 \%$ between the low-order model and the high-fidelity ANSYS simulation within the sheath. The maximum percent error for the axial stress for this cross-sectional cut is approximately $4 \%$ within the sheath. The increased error in the axial stress result comes from the addition of the axial stress due to the axial thermal expansion of the model. The radial and hoop stress analytical equations use the thick-walled cylindrical pressure equations, whereas the axial stress needs an additional simplified term in addition to the cylindrical pressure vessel equations adding to the error.
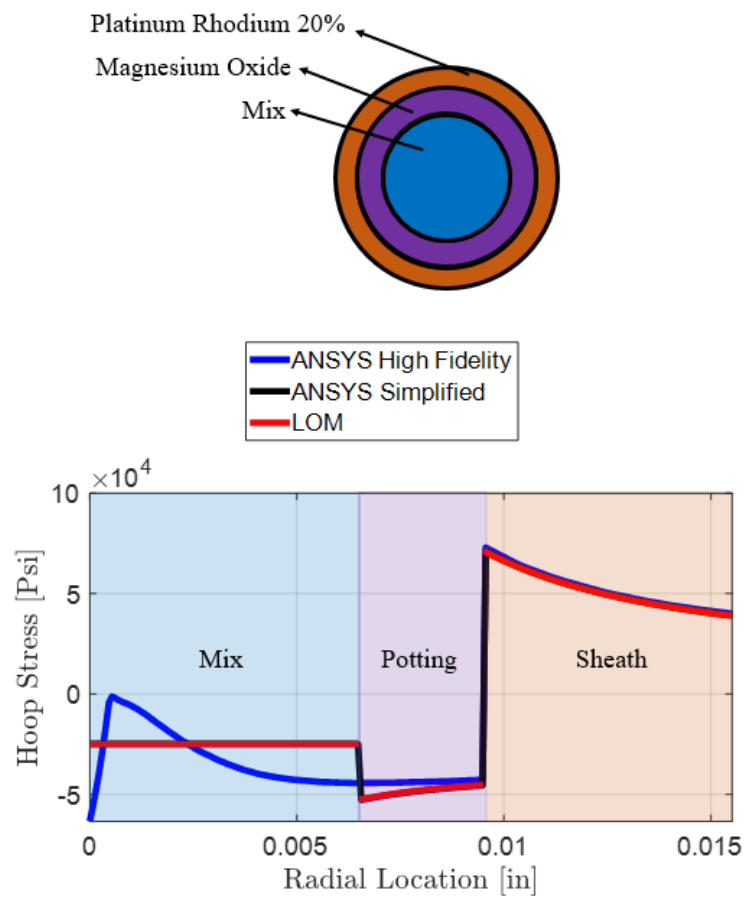
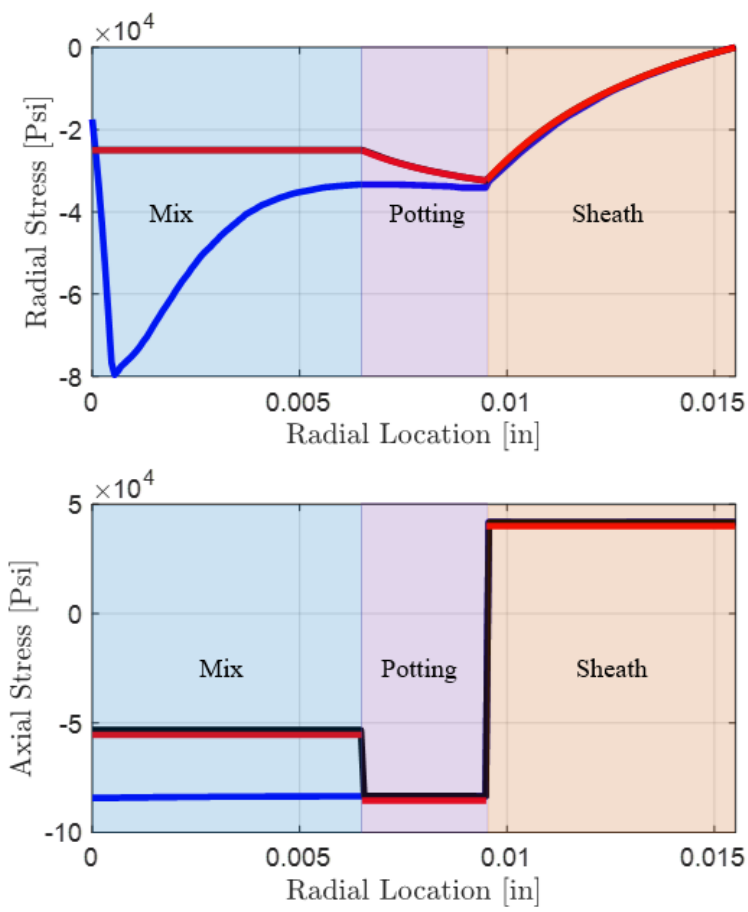

Fig. 20 Stress results from a cross-sectional cut in ANSYS and the LOM. 
Overall, the low-order model does an excellent job at approximating stresses throughout the sheath of the thermocouple in comparison with ANSYS mechanical, as can be seen in Fig. 21. Both ANSYS mechanical and the low-order model assume linearity so even if the stress value is beyond the yield point, the stress reported will be as if the structure never yields. This becomes very important when designing a probe for high temperatures while trying to keep a small geometry. The optimized design could potentially be right below the point of yielding.
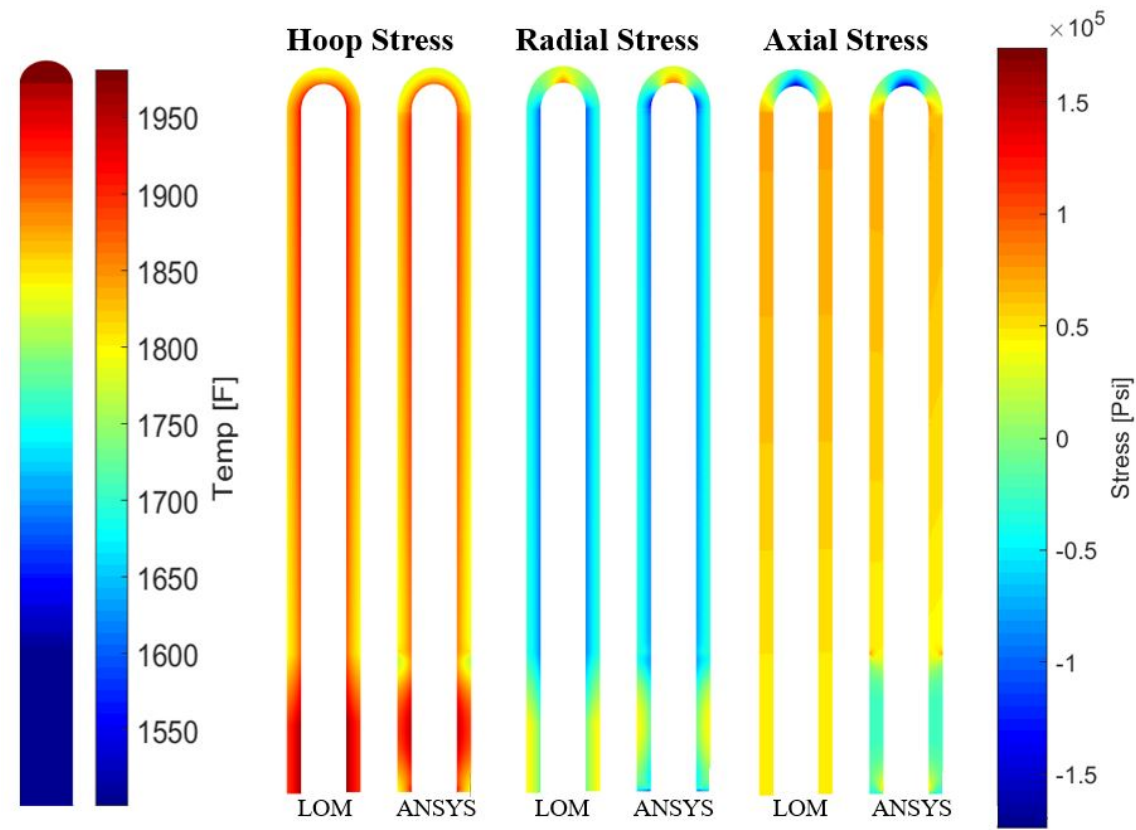

Fig. 21 Temperature profile and stress value comparison between ANSYS mechanical and the analytical low-order model in the sheath.

One thing to take note of is the temperature profile on the left-hand side of Fig. 21. This profile shows that in the base section the temperature is held constant at $1500^{\circ} \mathrm{F}\left(816^{\circ} \mathrm{C}\right)$ and the linear variation started reaching a maximum temperature of $2000^{\circ} \mathrm{F}\left(1093^{\circ} \mathrm{C}\right)$ in the tip. However, the linear temperature variation stops right where the main body of the thermocouple and the tip meet. This is because the analytical equations have no way of capturing the temperature variation within the spherical tip resulting in the tip temperature being constant. In reality, since the tip is solid and made out of one material it will be very close to the same temperature throughout, so this prediction remains a good approximation throughout the tip section.

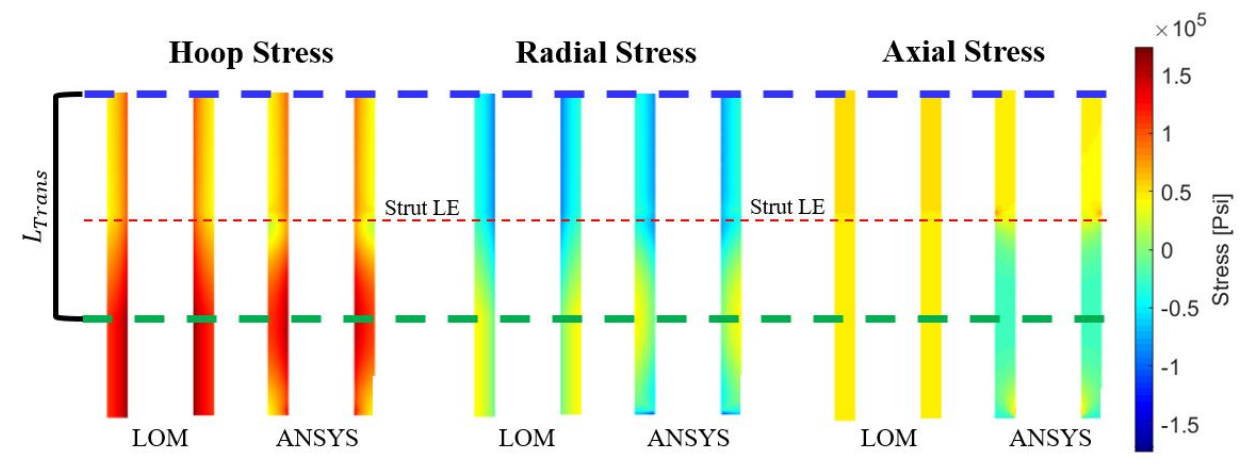

Fig. 22 Clamped base and transition zone stress results.

Referring back to Fig. 11, the clamped base is split into two parts. Below the green line, only the base analytical equations are used. Above the blue line, only the main body analytical equations are used. Between the two lines is a transition zone where both types of equations are used in a linear averaging scheme to get an approximate answer in that 
zone. Figure 22 proves that this procedure works. Two effects are skewing the results slightly. The first being that at the strut leading edge, right where the thermocouple exits the shield base, a stress concentration occurs in the ANSYS high-fidelity model. A stress singularity occurs because the edge of the shield face is perpendicular to the sheath of the thermocouple. In reality, this will never occur and a slight fillet will be present getting rid of the stress singularity. In this case, with the stress singularity, the region between the green line and the blue line within the low-order model more accurately reflects the physical world in comparison to ANSYS. The area below the green line within ANSYS is being affected by not modeling the entire thermocouple being fed through the strut. Instead, the no-displacement boundary condition has been placed at the base of the high-fidelity ANSYS model. The low-order model assumes that the base goes on forever and thus it does not produce the problems seen in the ANSYS stress contours. Again, in this specific case, the low-order model likely more accurately reflects the physical word.

Only hoop stress and radial stress within the base fall under the argument made in the previous paragraph. It is important to note that in the regions within the strut, the radial stress is positive and the hoop stress gets very large. This is due to the strut being made of Inconel 600. At the temperature chosen, the thermal expansion coefficient of Inconel 600 is larger than that of the magnesium oxide. This means that not only is the sheath being pushed from the inside by the expanding potting but also being pulled from the outside by the expanding strut.

As expected, the axial stress predicted by the low-order model within the shield base (below the red strut LE line) does not accurately reflect the axial stress predicted by the high-fidelity ANSYS simulations. Again, this is because when calculating the axial stress produced by the axial thermal expansion, plane strain is assumed. In the base region, the shield base and especially the equivalent strut cylinder have radii on the order of the entire length of the base section. Thus, the plane-strain assumption has been violated and will no longer accurately predict the stress. However, based on the high-fidelity ANSYS simulations, the axial stress in the base section is almost zero. This will always be the case within the sheath because the surrounding shield base and strut are much larger. Therefore, the axial stress within the base is of little importance.

The low-order model results for the main body of the thermocouple align with the high-fidelity ANSYS results almost perfectly as seen in Fig.23 . By visual inspection, the only stress that seems any different at all is the axial stress. The reason for this is that the two wires in a thermocouple are made of different materials. In this case, the platinum-rhodium $10 \%$ wire wants to expand more than the platinum-rhodium $30 \%$ wire. Over the long length of the thermocouple, the area-averaged mix inner rod in the simplified low-order model builds up error compared to the high-fidelity ANSYS simulation that is taking the effects of both wires into account. However, even with this error, the low-order model and ANSYS results compare very well to one another. In the case of hoop and radial stress, the maximum percent error is approximately $1 \%$, whereas in the axial stress case the maximum percent error is approximately $2.5 \%$ when compared to the high-fidelity simulation.

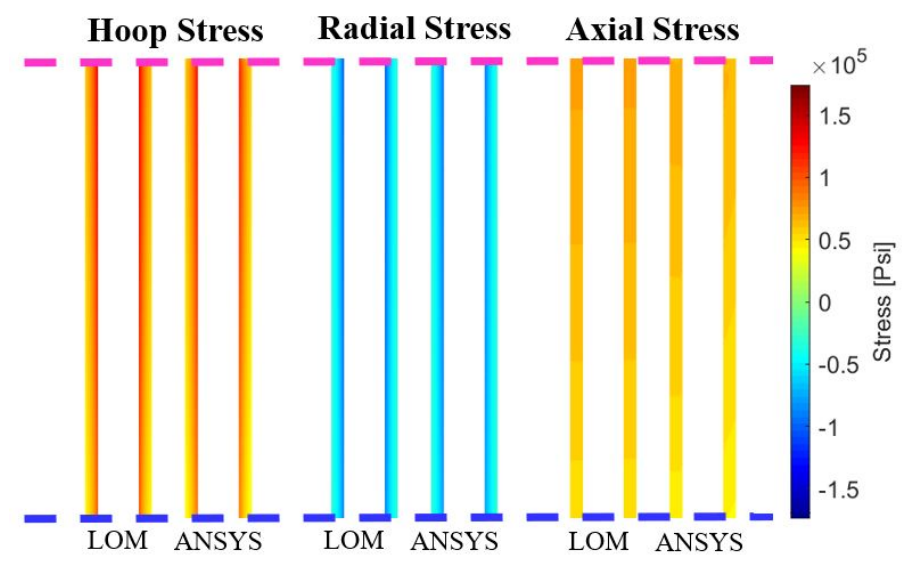

Fig. 23 Main body stress result comparisons.

As stated previously, analytically computing the stress within the tip is challenging for the simple fact that the spherical tip is connected to a large cylindrical body. Therefore, the tip stress equations must account for the effects felt by the large cylindrical main body. To do this, the main body equations and tip equations are combined using an angle-weighted, linear averaging scheme to get an approximate stress result. Figure 24 shows that this approach works well. Even though the stress gradients can be off by a wide margin in some places in the tip, the largest stress values 
and locations are captured accurately. In each of the three stress cases, the maximum percent error for both the largest positive stress and the largest negative stress is approximately $3 \%$. The maximum percent error in the entire tip is approximately $20 \%$ when compared to the high-fidelity simulation.

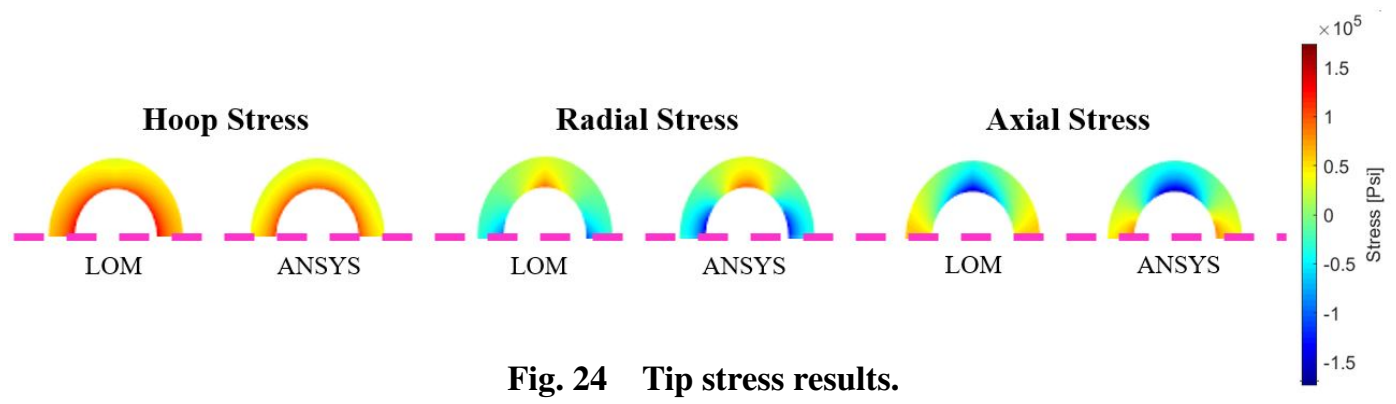

The combination of the cylindrical body with the spherical tip leads to complex stress gradients (Fig. 24). The spherical tip does not want to expand radially as much as the cylindrical main body does. As a result, after thermally expanding, the tip is no longer a dome shape. Instead, it is more of a half ellipsoid as shown in Fig. 25. The geometry change leads to the stress gradients seen in Fig. 23. As the tip is pulled into the ellipsoid shape, the radial stress at the very tip becomes positive as the tip is pulled in two directions by the cylindrical main body. This causes an increase in hoop stress as well. Since the cylindrical body also wants to deform more in the axial direction as well, the two halves of the tip (split left and right) are pushed up and apart from each other. This causes the axial stress to become negative at the very top of the tip.

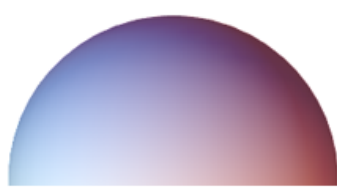

Pre-Expansion Tip

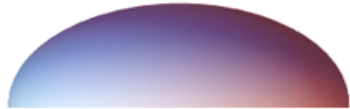

Post-Expansion Tip

Fig. 25 Exaggerated tip geometry change due to thermal expansion.

If a thermocouple of this type is to yield or fail, it will happen in either the clamped base region or in the tip. A likely source of yielding and eventual failure is on the vertical line (red line in Fig. 14). All three stresses have a maximum on the inner surface of the sheath along that line. Eventually, the thermal expansion of the main body region will impart so much stress on the tip region that the tip will yield along that line and will eventually be prone to failure.

\section{Comparison of Analytical and Experimental Results}

A simplified experiment was completed to compare the analytical low-order model to experimental results. The term "simplified" is used since a simple model was designed to act like a thermocouple at much lower temperatures (several hundred degrees) versus several thousand degrees like the comparison between the numerical and analytical models previously discussed. Instead of using an actual thermocouple that is very small, a large diameter brass rod was plated with nickel to produce the same kind of thermal expansion mismatch seen in high-temperature thermocouples at much lower temperatures. The thermal expansion coefficient of brass is about twice that of the nickel used. Since stress values cannot be directly measured, strain gauges were used to collect the strain on the outer surface of the rods. Doing this, allows the experimental results to be directly compared to those produced by the low-order model.

Figure 26 shows a comparison between the two rods built and a typical thermocouple configuration. The left side shows the "Large Rod" which has the 0.375 inch brass rod which has been plated with nickel to make the total radius approximately 0.6725 inch. This gives the large rod a thickness ratio of approximately 0.577 which is very comparable to the typical thickness ratio of 0.6 for a high-temperature thermocouple. The "Small Rod" has the same 0.375 inch brass rod in the center, but has half of the nickel plating thickness compared to the large rod. This gives the small rod a total diameter of approximately 0.5255 inch and a thickness ratio of approximately 0.713 . A higher thickness ratio results in a larger length over diameter ratio. Unfortunately, both rods had thicker plating than designed which resulted 
in different length to diameter ratios than intended. The large rod has a length to diameter ratio of approximately 7.5 while the small rod has a length to diameter ratio of approximately 12.5. As discussed before, the model assumes a length to diameter ratio of 10 . This means that the axial strain results for the large rod will not be directly comparable to those predicted by the low-order model. This will be discussed in further detail later.

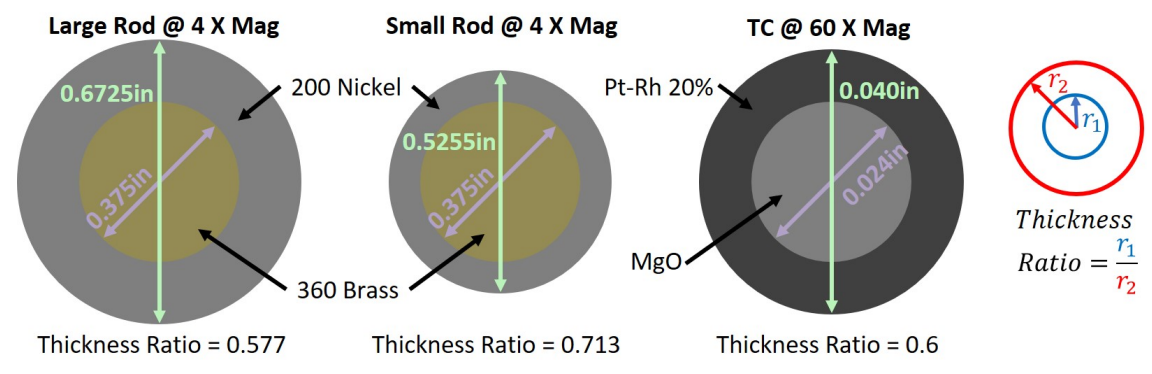

Fig. 26 Experimental rods Vs. typical thermocouple configuration. Magnification included for relative sizing.

Each rod was fitted with three bi-axial strain gauges (SK-06-015EH-120 and SK-06-015DJ-120 from MicroMeasurements) to measure strain on the outer surface in three different places as seen in Fig. 27. Each gauge was placed at different theta locations around the rod and approximately $1 \mathrm{inch}$ apart from each other in the axial direction. The gauges were selected to match the thermal expansion coefficient of the nickel so that any resulting strain would be due to the thermal expansion of the brass pushing against the nickel. The gauges were bonded to the surface using M-Bond 600, which is used for high temperatures up to $500^{\circ} \mathrm{F}$. This entire setup was then covered by a protective coating, Epoxylite 813 , to seal the electrical components, and that is also rated up to $500^{\circ} \mathrm{F}$ for long time periods. In total, each rod has a total of six strain gauges. Three gauges read axial strain and the other three read circumferential strain.

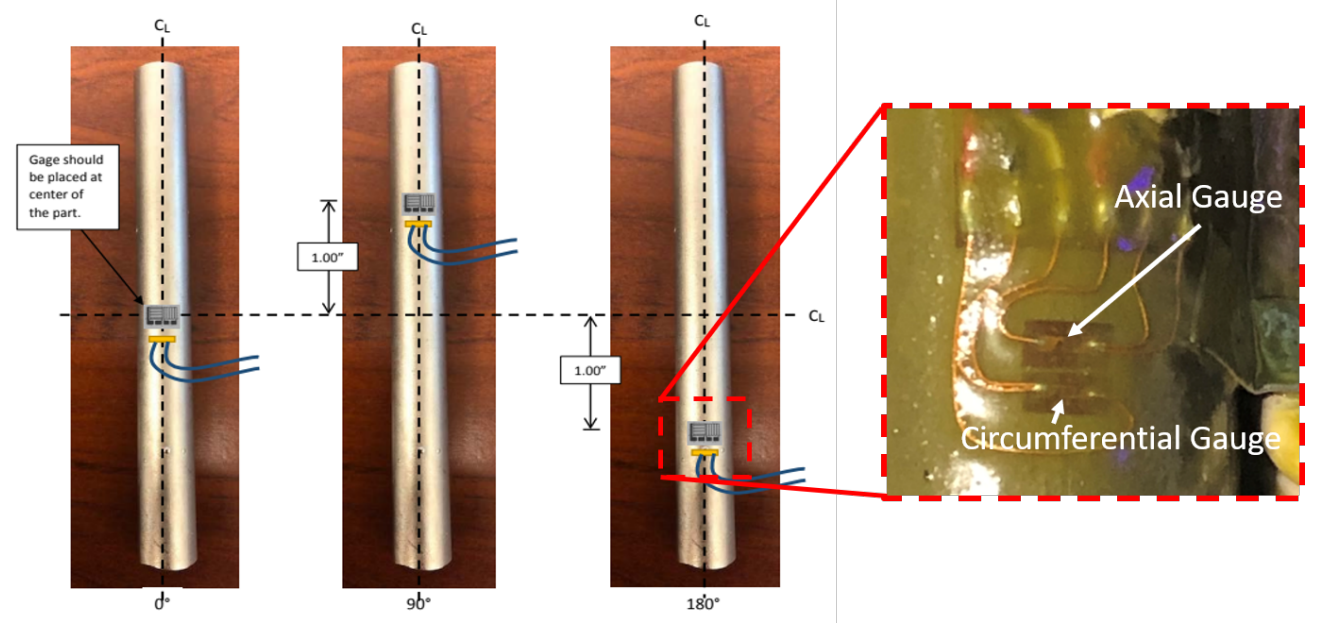

Fig. 27 Strain gauge placement.

These two rods were submerged in a Thermo Fisher Scientific SAHARA S7 (Fig. 28), stainless-steel, heated oil bath circulator and subject to temperatures up to $392^{\circ} \mathrm{F}\left(200^{\circ} \mathrm{C}\right)$. This oil bath was chosen as it can control the temperature to within $\pm 0.1^{\circ} \mathrm{C}$. It does this by constantly circulating the oil or in this case, silicone 180 , throughout the testing period. As stated previously, both rods were slightly larger in diameter than designed. This resulted in the predicted yield point being out of the range of the capability of what the oil bath could produce. This will be discussed in further detail later.

The rods were first submerged in the oil bath at room temperature so that a baseline could be taken and used as the zero strain point. From there, the temperature was increased in $25^{\circ} \mathrm{F}$ increments up to a maximum temperature of approximately $400^{\circ} \mathrm{F}$. As seen in Fig. 29, the rods took approximately 12 minutes between each increment to fully saturate in temperature and come to a steady-state voltage. The voltage was ultimately converted into a strain value. Data was then taken at the steady-state value for 30 seconds. The data for the 30 -second interval was then averaged to get a single data point which was compared to the low-order model. It can also be clearly seen that the smaller rod reacts 
to the temperature change much faster than the large rod which is expected since the temperature can saturate through the smaller radius faster than the larger radius of the large rod. All of the different colors in Fig. 29 represent individual strain gauges on each rod. In both rods, a voltage difference can be seen, especially in the small rod results. Looking at the small rod transient results, the dark purple is higher than the light blue. That is because the dark purple represents the circumferential strain and the light blue represents the axial strain. It will be shown later that the circumferential strain values were higher than the axial strain values, especially in the small rod. This finding aligns exactly with what is predicted by the low-order model.

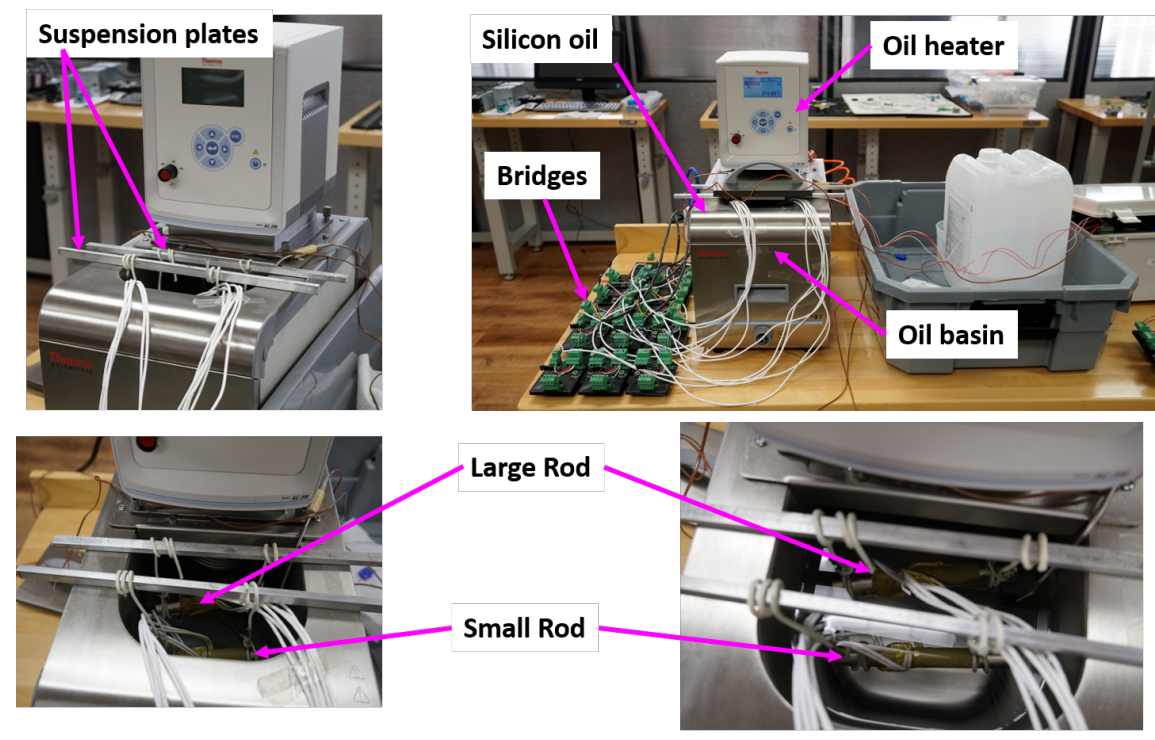

Fig. 28 Experimental setup.
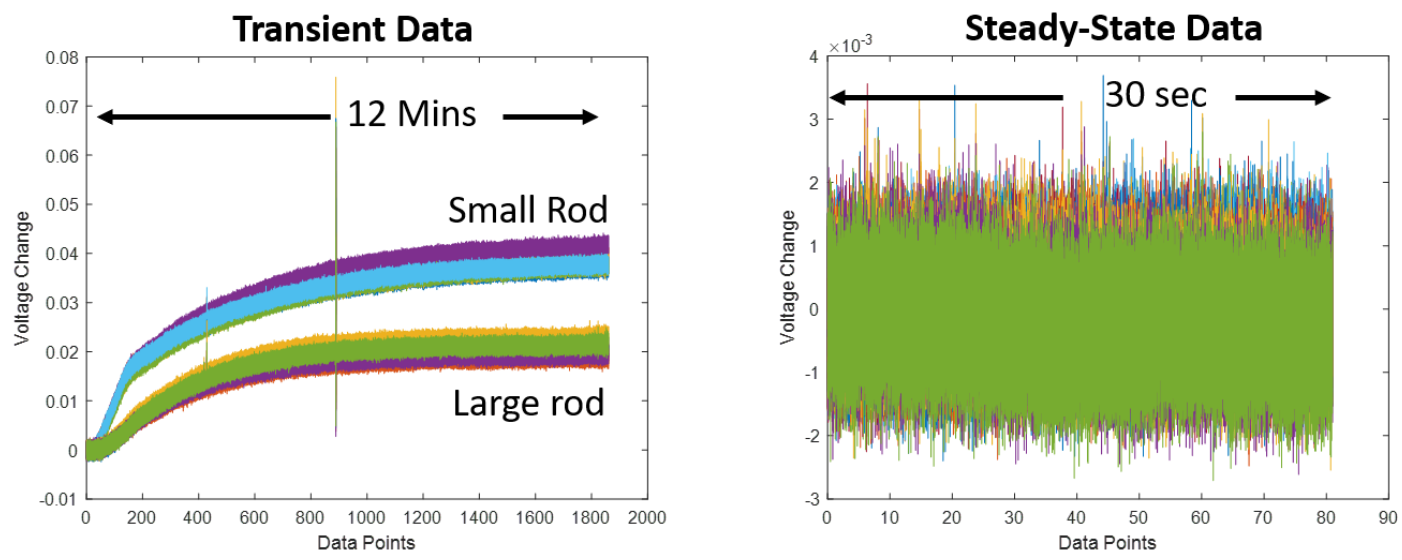

Fig. 29 Raw transient voltage data Vs. Steady-state voltage data.

It is important to note that the strain gauge values shown later in this section used a three-wire setup to account for the resistance change in the wiring with temperature. Comparing the two setups in Fig. 30 shows that in the two-wire setup if the resistance of the wires is changing then the Wheatstone bridge can become unbalanced and skew the results. However, the addition of another leadwire $\left(R_{L 3}\right)$ balances the Wheatstone bridge as the resistances change with temperature. This setup is absolutely crucial, since the experiment is designed to capture effects from changing temperatures. 


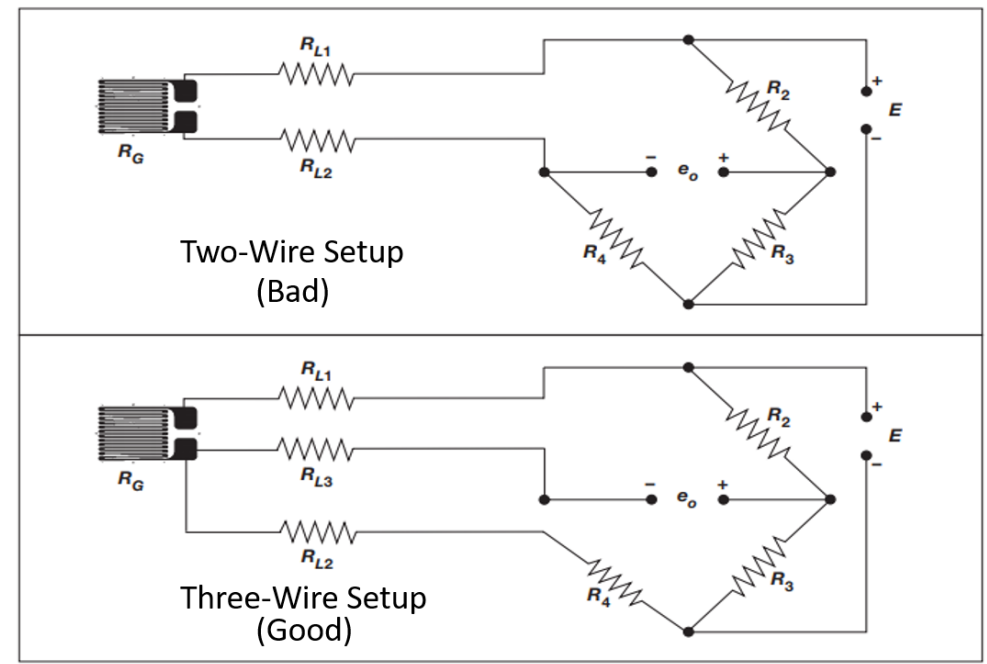

Fig. 30 Strain gauge wire setup.

Before the experimental data could be compared to the analytically predicted values, several strain corrections needed to be applied to the raw strain data. The first correction accounts for gauge factor variation with temperature. This correction factor becomes a multiplier to the raw data. Its equation can be seen below.

$$
\text { Gauge }_{F a c}=\frac{F^{*}}{F\left(T_{1}\right)}=\frac{F^{*}}{F_{\text {gauge }}\left(1+\frac{\Delta F(\%)}{100}\right)}
$$

Equation 33 consists of several variables. The first of which is $F^{*}$ which is the base gauge factor used by the supplying company to scale the actual gauge factor of the strain gauges. The second is $F_{\text {gauge }}$ which represents the actual gauge factor at room temperature of the strain gauges. $F_{\text {gauge }}$ is supplied by the provider with every strain gauge. Finally, the final value is $\Delta F(\%)$ which is the percentage of gauge factor change with temperature. Plotting Eq. 33 over the operating temperature range results in Fig. 31.

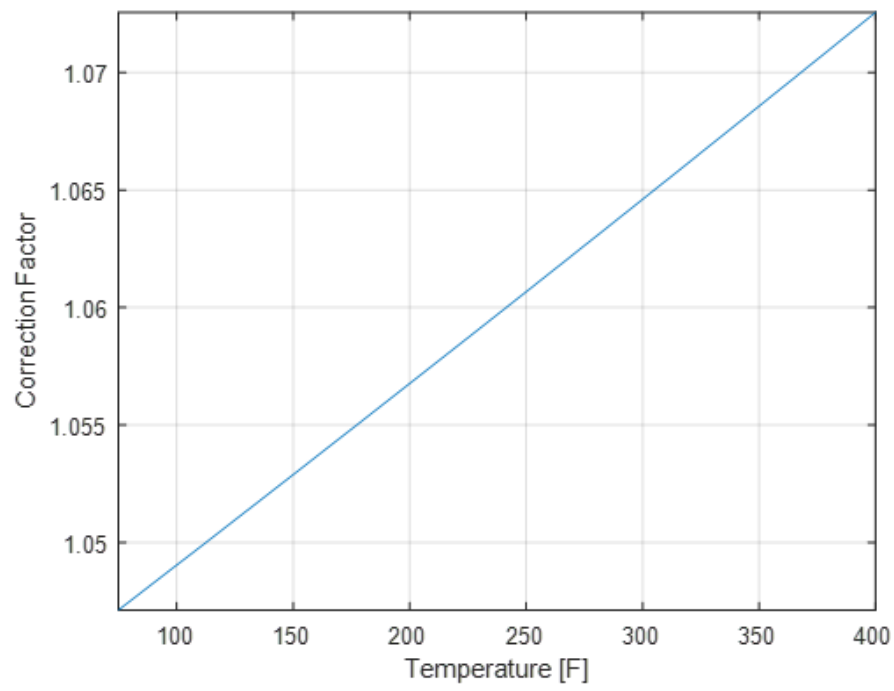

Fig. 31 Gauge factor correction variation with temperature. 
The second correction accounts for the curved surface on which the strain gauges were applied to. This correction also takes into account the effects of the adhesive and backing used to apply the strain gauge and hold it in place. This correction results in a strain value that must be added to the raw data before the gauge factor correction has been applied.

$$
\epsilon_{C u r v}=\frac{1}{R}\left[\left(1+2 v_{A-B}\right)\left(h_{A} \alpha_{A}+h_{B} \alpha_{B}\right)-2 v_{A-B} \alpha_{S}\left(h_{A}+h_{B}\right)\right] \Delta T
$$

Equation 34 contains several important variables. The first variable is $R$, which is the radius of curvature of the rod. This means this correction varies between the large rod and the small rod. The second variable is $v_{A-B}$, which is the average Poisson's ratio of the adhesive and backing of the strain gauge. Now looking at $h_{A}$ and $h_{B}$, these represent the adhesive and backing thickness, respectively. Finally, looking at $\alpha_{A}, \alpha_{B}$, and $\alpha_{S}$ these represent the thermal expansion coefficient of the adhesive, backing, and substrate, respectively. The resulting strain correction curve has been calculated for both the small and large rods. The strain correction curve for the small rod is shown in Fig. 32.

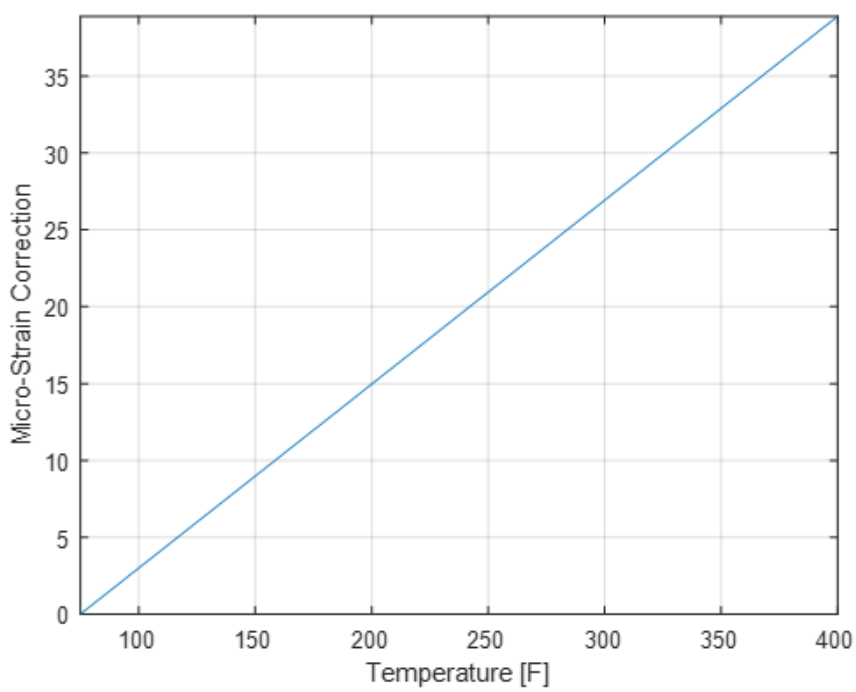

Fig. 32 Curvature strain correction variation with temperature.

Putting both corrections together results in Eq. 35 which is the corrected strain value equation used to transform the raw data into the data that will be compared with the low-order model (Fig. 33).

$$
\epsilon_{\text {Corrected }}=\left(\epsilon_{\text {Raw }}+\epsilon_{\text {Curv }}\right) * \text { Gauge } e_{F a c}
$$

Looking to the left side of Fig. 33, the small rod analytical predictions for the circumferential strain is plotted in a continuous blue line while the axial strain is plotted in red. The results from the strain gauges are also plotted with each gauge having a specific shape while the colors refer to circumferential or axial strain. The small rod results clearly show good agreement between the analytical predictions and the experimental data. The maximum absolute percent error in circumferential strain is approximately $7 \%$, while the maximum absolute percent error in axial strain is approximately $9.5 \%$. This occurs at the lower temperatures. The reason for this is that the oil bath was much more controllable at temperatures above $200^{\circ} \mathrm{F}$. Some fluctuation of temperature was seen below $200^{\circ} \mathrm{F}$, and the sensors were very sensitive to this. A fluctuation of $5^{\circ} \mathrm{F}$ could lead to a strain difference of 10 micro-strain. This type of possible error is on the same order of magnitude as that seen in the experimental data. Overall, the small rod satisfies all assumptions necessary for comparison with the low-order model and produces a very good comparison.

Looking at the right side of Fig. 33, the large rod again plots the analytical predictions for circumferential strain in a continuous blue line while the axial strain is plotted in red.The circumferential strain aligns very well with the predicted data. However, the axial strain experimental results do not align well with the analytical predictions. This arises for the small length to diameter ratio of 7.5. Again, the model assumes this ratio to be at least 10 . The result of this is that 
while the circumferential strain has a maximum absolute percent error of around 5\%, the axial strain ends up having a maximum absolute percent error of nearly $43 \%$. This result shows just how crucial it is to understand the assumptions made in the model to get accurate results.
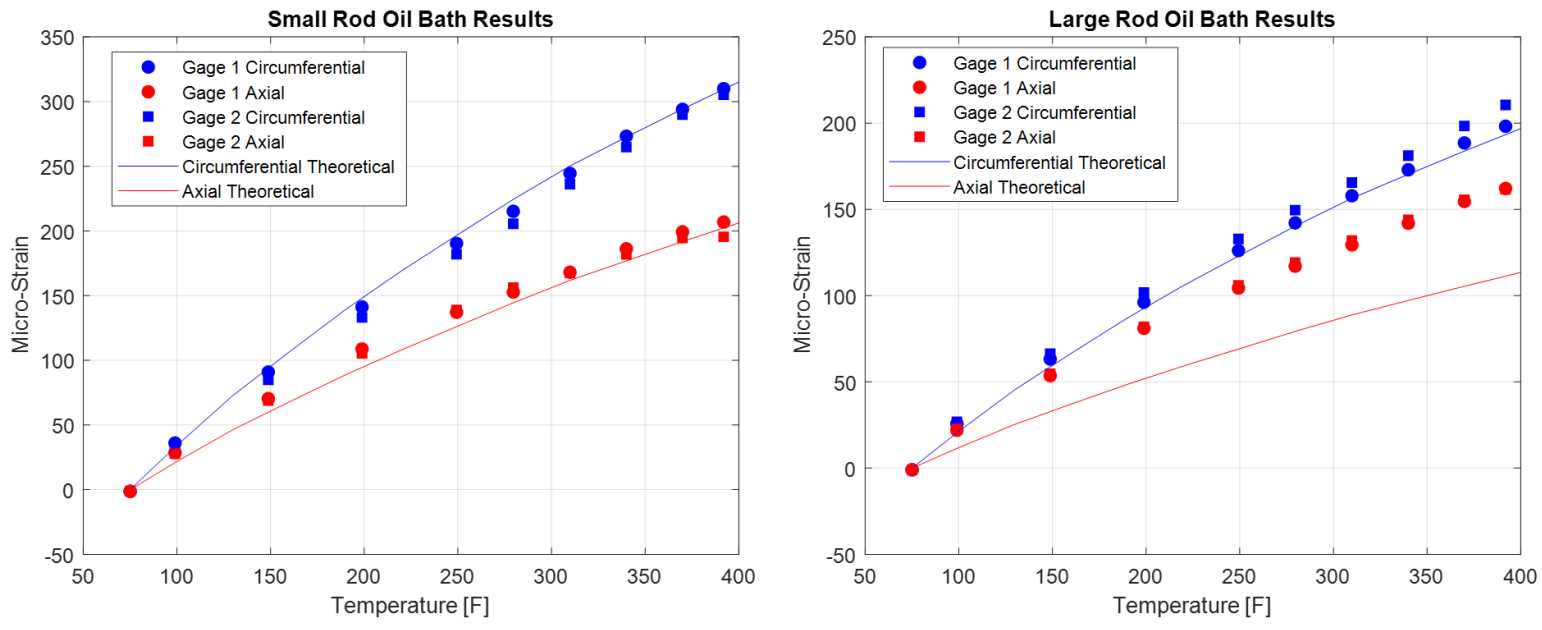

Fig. 33 Experimental results comparison with analytical predictions.

\section{Conclusions}

In this study, the structural behavior of a sheathed noble metal thermocouple at high temperatures was investigated using a newly-developed analytical low-order model validated against high-fidelity ANSYS simulations and a simplified experiment. A new analytical low-order model was developed with several simplifying assumptions. These assumptions made it possible to simplify the geometry and constraints of the problem sufficiently enough to derive quasi 3D analytical equations that represent the stresses within the sheath of a thermocouple in an accurate way.

A caveat to this approach is that the potting and wire stress values are not accurate when using the low-order model. Since both wires and potting are under compression in every part of the thermocouple their stress values are inconsequential compared to their compressive strengths. Buckling is of no concern since the potting is already in powdered form and the wires want to expand at relatively the same rate as the sheath. Several key results were noted:

1) An analytical low-order model using quasi $3 D$ equations represents the stresses in the sheath of a thermocouple well but not in the potting or the wires due to the simplifying assumptions used. The sheath is the most critical region.

2) High stress in the sheath results from using a sheath material that has a lower thermal expansion coefficient than the potting.

3) Stress concentration regions can occur at the base of the thermocouple depending on the materials that the thermocouple is mounted into.

4) A length to diameter ratio of at least 10 is needed to satisfy the low-order model assumptions.

5) Large stress concentrations occur near the tip of the sheath because of the complex interactions between the spherical tip and the cylindrical main body.

An analytical model will always have the advantage of computation speed over a numerical model. In the world of rapid development and optimization, an analytical model saves both time and money. For example, the high-fidelity ANSYS simulation presented in this study took over twenty-two hours to run. However, the low-order model takes approximately six seconds to get within acceptable accuracy compared to the high-fidelity simulation in a large majority of the thermocouple's sheath. That means that the low-order model is over 13,000 times faster than the equivalent high-fidelity ANSYS simulation. This kind of time and cost savings has a large impact on the world of design optimization. 
Overall, through the use of quasi 3D developed analytical equations and weighted linear averaging of those equations, it is possible to produce a low-order analytical model that compares well with a high-fidelity ANSYS mechanical simulation and experiment values in the majority of the thermocouple. The implementation of this in conjunction with a thermal performance low-order model such as that presented in Ref. [1] creates a powerful tool for use in a fluid-structure interaction rapid design space that optimizes the design and saves a large amount of time and money.

The low-order model can rapidly provide probe designs that can be weighted based on strength and thermal performance and compared with one another to achieve the highest strength probe based on the analytical results. This analytically optimized probe could then be modeled within ANSYS mechanical to ensure the stress values are in agreement. For example, in two days a validated optimized probe design for structural needs could be developed, whereas if the same amount of thermocouple designs were run through ANSYS mechanical it would have taken years.

\section{Appendix}

Table 2 Magnesium Oxide material properties [11, 12].

\begin{tabular}{||cccc||}
\hline$T[\mathrm{~K}]$ & $\alpha\left[\mathrm{K}^{-1}\right]$ & $E[\mathrm{~Pa}]$ & $v$ \\
\hline 200 & - & $2.8094 \mathrm{E} 11$ & 0.2170 \\
250 & - & $2.7896 \mathrm{E} 11$ & 0.2178 \\
300 & $10.42 \mathrm{E}-6$ & $2.7682 \mathrm{E} 11$ & 0.2194 \\
350 & $11.22 \mathrm{E}-6$ & $2.7456 \mathrm{E} 11$ & 0.2203 \\
400 & $12.02 \mathrm{E}-6$ & $2.722 \mathrm{E} 11$ & 0.2211 \\
450 & $12.47 \mathrm{E}-6$ & $2.6983 \mathrm{E} 11$ & 0.2220 \\
500 & $12.91 \mathrm{E}-6$ & $2.6740 \mathrm{E} 11$ & 0.2228 \\
550 & $13.17 \mathrm{E}-6$ & $2.6493 \mathrm{E} 11$ & 0.2237 \\
600 & $13.42 \mathrm{E}-6$ & $2.6244 \mathrm{E} 11$ & 0.2245 \\
650 & $13.66 \mathrm{E}-6$ & $2.5993 \mathrm{E} 11$ & 0.2254 \\
700 & $13.90 \mathrm{E}-6$ & $2.5741 \mathrm{E} 11$ & 0.2263 \\
750 & $14.10 \mathrm{E}-6$ & $2.5487 \mathrm{E} 11$ & 0.2271 \\
800 & $14.30 \mathrm{E}-6$ & $2.5232 \mathrm{E} 11$ & 0.2280 \\
850 & $14.43 \mathrm{E}-6$ & - & - \\
900 & $14.55 \mathrm{E}-6$ & $2.4719 \mathrm{E} 11$ & 0.2298 \\
950 & $14.68 \mathrm{E}-6$ & - & - \\
1000 & $14.81 \mathrm{E}-6$ & $2.4202 \mathrm{E} 11$ & 0.2317 \\
1050 & $14.96 \mathrm{E}-6$ & - & - \\
1100 & $15.11 \mathrm{E}-6$ & $2.3687 \mathrm{E} 11$ & 0.2336 \\
1150 & $15.28 \mathrm{E}-6$ & - & - \\
1200 & $15.45 \mathrm{E}-6$ & $2.3168 \mathrm{E} 11$ & 0.2355 \\
1250 & $15.60 \mathrm{E}-6$ & - & - \\
1300 & - & $2.2648 \mathrm{E} 11$ & 0.2375 \\
1400 & - & $2.2128 \mathrm{E} 11$ & 0.2395 \\
1500 & - & $2.1606 \mathrm{E} 11$ & 0.2417 \\
1600 & - & $2.1084 \mathrm{E} 11$ & 0.2439 \\
\hline
\end{tabular}


Table 3 Inconel 600 material properties [8].

\begin{tabular}{||cccc||}
\hline$T[\mathrm{~K}]$ & $\alpha\left[\mathrm{K}^{-1}\right]$ & $E[\mathrm{~Pa}]$ & $v$ \\
\hline 223.15 & $12.3 \mathrm{E}-6$ & - & - \\
293.15 & $10.4 \mathrm{E}-6$ & - & - \\
295.15 & - & $2.14 \mathrm{E} 11$ & 0.324 \\
373.15 & $13.3 \mathrm{E}-6$ & $2.10 \mathrm{E} 11$ & 0.319 \\
473.15 & $13.8 \mathrm{E}-6$ & $2.05 \mathrm{E} 11$ & 0.314 \\
573.15 & $14.2 \mathrm{E}-6$ & $1.99 \mathrm{E} 11$ & 0.306 \\
673.15 & $14.5 \mathrm{E}-6$ & $1.93 \mathrm{E} 11$ & 0.301 \\
773.15 & $14.9 \mathrm{E}-6$ & $1.87 \mathrm{E} 11$ & 0.300 \\
873.15 & $15.3 \mathrm{E}-6$ & $1.80 \mathrm{E} 11$ & 0.301 \\
973.15 & $15.8 \mathrm{E}-6$ & $1.72 \mathrm{E} 11$ & 0.305 \\
1173.15 & $16.1 \mathrm{E}-6$ & $1.64 \mathrm{E} 11$ & 0.320 \\
1273.15 & $16.4 \mathrm{E}-6$ & $1.54 \mathrm{E} 11$ & 0.330 \\
1373.15 & - & $1.43 \mathrm{E} 11$ & 0.339 \\
\hline
\end{tabular}

Table 4 Platinum-rhodium $30 \%$ material properties $[9,10]$.

\begin{tabular}{||cccc||}
\hline$T[\mathbf{K}]$ & $\alpha\left[\mathbf{K}^{-1}\right]$ & $E[\mathrm{~Pa}]$ & $v$ \\
\hline 298.15 & - & $2.777 \mathrm{E} 11$ & 0.324 \\
373.15 & $9.20 \mathrm{E}-6$ & - & - \\
473.15 & $8.50 \mathrm{E}-6$ & $2.657 \mathrm{E} 11$ & 0.330 \\
573.15 & $9.00 \mathrm{E}-6$ & - & - \\
673.15 & $8.75 \mathrm{E}-6$ & $2.510 \mathrm{E} 11$ & 0.334 \\
773.15 & $9.00 \mathrm{E}-6$ & $2.439 \mathrm{E} 11$ & 0.338 \\
873.15 & $9.00 \mathrm{E}-6$ & $2.366 \mathrm{E} 11$ & 0.340 \\
973.15 & $9.14 \mathrm{E}-6$ & $2.295 \mathrm{E} 11$ & 0.343 \\
1073.15 & $9.38 \mathrm{E}-6$ & $2.221 \mathrm{E} 11$ & 0.345 \\
1173.15 & $9.44 \mathrm{E}-6$ & $2.157 \mathrm{E} 11$ & 0.346 \\
1273.15 & $9.70 \mathrm{E}-6$ & $2.093 \mathrm{E} 11$ & 0.35 \\
1373.15 & $9.91 \mathrm{E}-6$ & $2.028 \mathrm{E} 11$ & 0.352 \\
1473.15 & $10.08 \mathrm{E}-6$ & $1.954 \mathrm{E} 11$ & 0.358 \\
1573.15 & $10.38 \mathrm{E}-6$ & - & - \\
1673.15 & $10.64 \mathrm{E}-6$ & - & - \\
1773.15 & $11.00 \mathrm{E}-6$ & - & - \\
\hline
\end{tabular}


Table 5 Platinum-rhodium $20 \%$ material properties $[9,10]$.

\begin{tabular}{||cccc||}
\hline$T[\mathbf{K}]$ & $\alpha\left[\mathbf{K}^{-1}\right]$ & $E[\mathrm{~Pa}]$ & $v$ \\
\hline 298.15 & - & $2.459 \mathrm{E} 11$ & 0.342 \\
373.15 & $6.30 \mathrm{E}-6$ & - & - \\
473.15 & $7.00 \mathrm{E}-6$ & $2.366 \mathrm{E} 11$ & 0.346 \\
573.15 & $7.66 \mathrm{E}-6$ & - & - \\
673.15 & $8.00 \mathrm{E}-6$ & $2.247 \mathrm{E} 11$ & 0.351 \\
773.15 & $8.60 \mathrm{E}-6$ & $2.188 \mathrm{E} 11$ & 0.353 \\
873.15 & $8.83 \mathrm{E}-6$ & $2.130 \mathrm{E} 11$ & 0.355 \\
973.15 & $9.0 \mathrm{E}-6$ & $2.072 \mathrm{E} 11$ & 0.358 \\
1073.15 & $9.38 \mathrm{E}-6$ & $2.010 \mathrm{E} 11$ & 0.359 \\
1173.15 & $9.66 \mathrm{E}-6$ & $1.955 \mathrm{E} 11$ & 0.360 \\
1273.15 & $9.90 \mathrm{E}-6$ & $1.898 \mathrm{E} 11$ & 0.362 \\
1373.15 & $10.18 \mathrm{E}-6$ & $1.846 \mathrm{E} 11$ & 0.367 \\
1473.15 & $10.42 \mathrm{E}-6$ & $1.792 \mathrm{E} 11$ & 0.380 \\
1573.15 & $10.62 \mathrm{E}-6$ & - & - \\
1673.15 & $10.86 \mathrm{E}-6$ & - & - \\
1773.15 & $11.13 \mathrm{E}-6$ & - & - \\
\hline
\end{tabular}

Table 6 Platinum-rhodium $10 \%$ material properties $[9,10]$.

\begin{tabular}{||cccc||}
\hline$T[\mathrm{~K}]$ & $\alpha\left[\mathrm{K}^{-1}\right]$ & $E[\mathrm{~Pa}]$ & $v$ \\
\hline 298.15 & - & $2.126 \mathrm{E} 11$ & 0.365 \\
373.15 & $10.00 \mathrm{E}-6$ & - & - \\
473.15 & $10.00 \mathrm{E}-6$ & $2.063 \mathrm{E} 11$ & 0.368 \\
573.15 & $10.00 \mathrm{E}-6$ & - & - \\
673.15 & $10.25 \mathrm{E}-6$ & $1.979 \mathrm{E} 11$ & 0.372 \\
773.15 & $10.2 \mathrm{E}-6$ & $1.933 \mathrm{E} 11$ & 0.376 \\
873.15 & $10.17 \mathrm{E}-6$ & $1.887 \mathrm{E} 11$ & 0.376 \\
973.15 & $10.29 \mathrm{E}-6$ & $1.839 \mathrm{E} 11$ & 0.378 \\
1073.15 & $10.38 \mathrm{E}-6$ & $1.792 \mathrm{E} 11$ & 0.379 \\
1173.15 & $10.44 \mathrm{E}-6$ & $1.750 \mathrm{E} 11$ & 0.383 \\
1273.15 & $10.60 \mathrm{E}-6$ & $1.697 \mathrm{E} 11$ & 0.381 \\
1373.15 & $10.64 \mathrm{E}-6$ & $1.649 \mathrm{E} 11$ & 0.385 \\
1473.15 & $10.92 \mathrm{E}-6$ & - & - \\
1573.15 & $11.08 \mathrm{E}-6$ & - & - \\
1673.15 & $11.29 \mathrm{E}-6$ & - & - \\
1773.15 & $11.73 \mathrm{E}-6$ & - & - \\
\hline
\end{tabular}


Table 7 Airfoil profile. [13].

\begin{tabular}{||ccc||}
\hline$X$ & $Y$ & $Z$ \\
\hline 1 & 0 & 0 \\
0.95 & 0.00707 & 0 \\
0.9 & 0.01259 & 0 \\
0.8 & 0.02099 & 0 \\
0.7 & 0.02635 & 0 \\
0.6 & 0.02917 & 0 \\
0.5 & 0.03 & 0 \\
0.4 & 0.02927 & 0 \\
0.3 & 0.02709 & 0 \\
0.2 & 0.02332 & 0 \\
0.1 & 0.01729 & 0 \\
0.075 & 0.01516 & 0 \\
0.05 & 0.01255 & 0 \\
0.025 & 0.00903 & 0 \\
0.0125 & 0.00646 & 0 \\
0 & 0 & 0 \\
0.0125 & -0.00646 & 0 \\
0.025 & -0.00903 & 0 \\
0.05 & -0.01255 & 0 \\
0.075 & -0.01516 & 0 \\
0.1 & -0.01729 & 0 \\
0.15 & -0.02067 & 0 \\
0.2 & -0.02332 & 0 \\
0.3 & -0.02709 & 0 \\
0.4 & -0.02927 & 0 \\
0.5 & -0.03 & 0 \\
0.6 & -0.02917 & 0 \\
0.7 & -0.02635 & 0 \\
0.8 & -0.02099 & 0 \\
0.9 & -0.01259 & 0 \\
0.95 & -0.00707 & 0 \\
1 & 0 & 0 \\
\hline & & \\
0
\end{tabular}

\section{Acknowledgments}

The authors gratefully acknowledge the support for this work, in addition to many helpful and insightful technical discussions, provided by Pratt \& Whitney through the Center of Excellence at Virginia Polytechnic Institute and State University. The authors would also like to acknowledge the support provided by Ahmic Aerospace through many insightful discussions and technical work to complete the experiment. 


\section{References}

[1] Vincent, T. G., "Total Temperature Probe Performance for Subsonic Flows using Mixed Fidelity Modeling," Ph.D. Dissertation, Virginia Tech 2019.

[2] "How to choose a Thermocouple," https://www.omega.com/en-us/ Available: https://www.omega.com/en-us/resources/selectinga-thermocouple.

[3] “Thermocouple Primer,” Thermocouple Primer - Phidgets Support Available: https://www.phidgets.com/docs/Thermocouple Primer.

[4] Hibbeler, R. C., and Yap, K. B., Mechanics of materials, Harlow, United Kingdom: Pearson, 2018.

[5] Slaughter, W. S., The linearized theory of elasticity: with 153 figures, New York: Springer Science Business Media, LLC, 2013.

[6] Shames, I. H., and Dym, C. L., Energy and Finite Element Methods in Structural Mechanics, New York, NY: Taylor Francis, 2003.

[7] “Stresses in thin-walled Pressure Vessels," Arveng Training Engineering Available: https://arvengtraining.com/en/stresses-inthin-walled-pressure-vessels/.

[8] "Inconel 600 Alloy Database" Available: http://www.specialmetals.com/assets/smc/documents/alloys/inconel/inconel-alloy600.pdf.

[9] Merker, J., Lupton, D., Topfer, M., and Knake, H., "High Temperature Mechanical Properties of the Platinum Group Metals Elastic Properties of Platinum, Rhodium and Iridium and Their Alloys at High Temperatures," Johnson Matthey Technology Review, vol. 45, 2001, pp. 74-82.

[10] Barter, B., and Darling, A. S., "Thermal Expansion of Rhodium-Platinum Alloys,” Johnson Matthey Technology Review, vol. 4, p. 138.

[11] Madhusudhan Rao, A. S., and Narender, K., "Studies on Thermophysical Properties of CaO and MgO by Gamma-Ray Attenuation," Journal of Thermodynamics, vol. 2014, Apr. 2014, pp. 1-8.

[12] Soga, N., and Anderson, O. L., "High-Temperature Elastic Properties of Polycrystalline MgO and Al2O3," Journal of the American Ceramic Society, vol. 49, May 1966, pp. 355-359.

[13] Bensiger, S. S. B., and Prasanth, N., "Analysis of Bi-Convex Aerofoil using CFD Software at Supersonic and Hypersonic Speed,” Elixir International Journal, Nov. 2012, pp. 2-2. 


\section{Chapter 3}

\section{Conclusions and Outlook}

The present work focused on two main areas necessary for advancing the development of high-temperature thermocouples through low-order analytical modeling. The first, and main area, focused on understanding how thermal expansion affects the stress within the metal sheath of a thermocouple at varying high temperatures. In addition, varying material combinations were also studied and put into two main categories of which only one category was classified as a problem. The second area, which is only sparsely discussed in this Thesis, focused on the combination of the previously developed thermal low-order models[1] and the newly developed structural low-order model. The combination of these two leads to a more complete low-order modeling package that is invaluable for a designer interested in accurate large-scale trade studies.

\subsection{Conclusions}

The structural behavior of a sheathed, noble-metal thermocouple at extreme temperatures was investigated using a newly-developed analytical low-order model validated against high-fidelity ANSYS Mechanical simulations and an idealized experiment. The analytical low-order model was developed employing several simplifying geometric assumptions. These assumptions simplified the model sufficiently to derive quasi-3D analytical equations that represent the stresses within the sheath of a thermocouple in an accurate manner.

This kind of quasi-3D analytical approach provides a large time advantage over numerical simulations. However, a caveat to this simplified approach is that the potting and wire stress values are not accurate within the low-order model. The combination of both wires and potting into the middle, area-averaged region has essentially smeared out all of the complex interactions between these components. However, the stress values seen in the fully modeled numerical simulations are very small in these areas compared to those in the sheath 
and are, therefore, of little concern. Another caveat is the stipulation that the length to diameter ratio of the probe must be at least ten for the quasi-3D equations to be reasonably accurate. Most thermocouples in practical applications satisfy this condition.

Focusing on the sheath, it was discovered that the high stress in the sheath resulted from a thermal expansion mismatch between the noble-metal sheath and the powdered ceramic insulation. All commonly used high-temperature thermocouples have a higher thermal expansion coefficient in the insulation compared to the metal sheath which turns the thermocouple into a thick-walled pressure vessel. An extension of this problem is seen in the base of the thermocouples where they are mounted. Large stress concentrations can show up in this region depending on the material of the mount. If its thermal expansion coefficient is larger than that of the sheath, then the stress is increased in the sheath. However, if the thermal expansion coefficient of the mount is less than that of the sheath, the stress is reduced within the sheath. Finally, large stress concentrations occur near the tip of the thermocouple because of the complex interactions between the spherical tip, cylindrical main body, and the presence of a thermocouple bead just below the tip in the case of an ungrounded probe.

This low-order model will always have an advantage in computation speed over numerically derived models. In the world of rapid development and optimization, a low-order analytical model saves time and money. For example, if the same model is run through both the structural low-order model and ANSYS Mechanical, the structural low-order model is approximately four orders of magnitude faster. This kind of time advantage provides the capability to rapidly produce and analyze probe designs so that large-scale trade studies and sensitivity analysis can be performed. This capability allows for highly optimized probe designs for specific uses to be created with high confidence in the same amount of time it would take to run one numerical simulation. Ultimately, the combination of the structural loworder model and the thermal low-order model previously developed[1] creates an unmatched capability to rapidly and accurately produce the best probe possible for the environment that it will be installed in.

S. Powers 2020 


\subsection{Outlook}

Several key structural topics remain to be studied to fully characterize thermocouple probe survival and performance under high-temperature conditions. The most pressing of these being structural dynamics (see Fig. 3.1). Due to the thermocouple being exposed to a high-speed flow, vibration will surely occur. Since thermocouples are generally mounted by the base, they will act just like a cantilevered beam. The second major piece of structural dynamics would be crack formation and growth. As the thermocouple heats up, the largest stresses are seen on the inside surface of the metal sheath. If any imperfections are present and the stress gets high enough, a crack would likely form. As the temperature continued to increase the crack would get larger and larger. However, thermal expansion, vibration, and crack growth are coupled together. Taking these three into account gives a more complete picture of the failure modes that could be expected in extreme conditions.

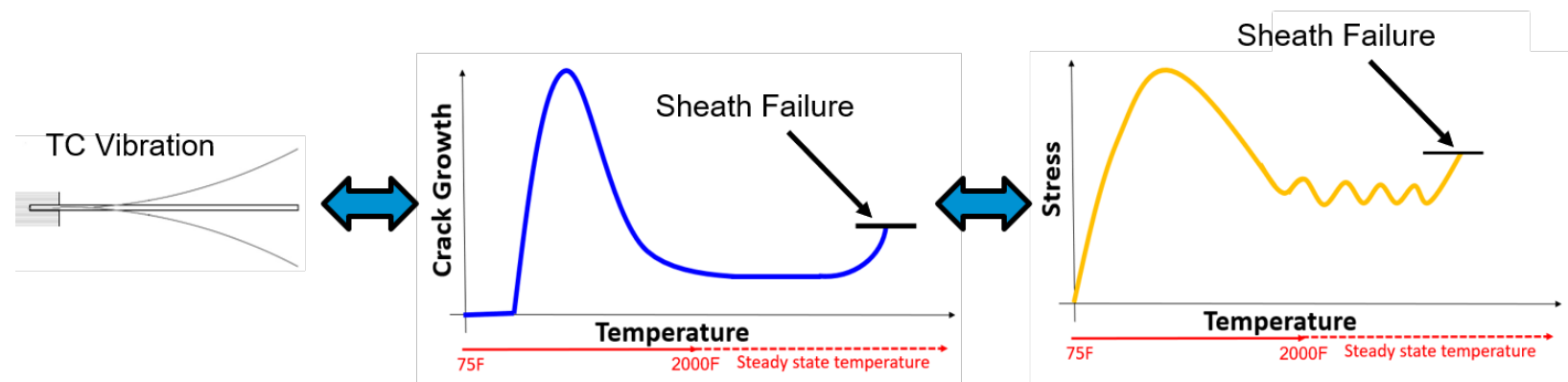

Figure 3.1: Structural Dynamics of Extreme Environment Thermocouples.

As illustrated in Fig. 3.1, as the probe is exposed to the flow it will start to vibrate starting at the base. The probe's temperature will also begin to increase. This initial flow exposure causes the stress within the sheath to rise rapidly. This will lead to small cracks being formed around the inside surface of the sheath. As the temperature continues increasing, the crack will grow, and the stress will initially decrease. As this continues, a cyclic process occurs where the sheath cracks, the stress goes down, the temperature continues to increase, stress goes up, sheath cracks more, etc. This is compounded by the vibrational effects. Eventually, the temperature of the flow (and thermocouple) reaches steady-state values and crack growth is no longer fueled by the thermal expansion coefficient mismatch. Crack growth is now only driven by vibrational effects. It should be noted that 
since the cracks have formed in the sheath it is likely that it will have lost some of its stiffness and thus increased the stress due to vibration. Over time, the vibration will cause the cracks to grow. Eventually, the cracks will break through the sheath at which point the sheath is considered to have failed. The period over which this takes place is highly reliant on flow characteristics, thermal expansion coefficient mismatch, and the thickness of the sheath.

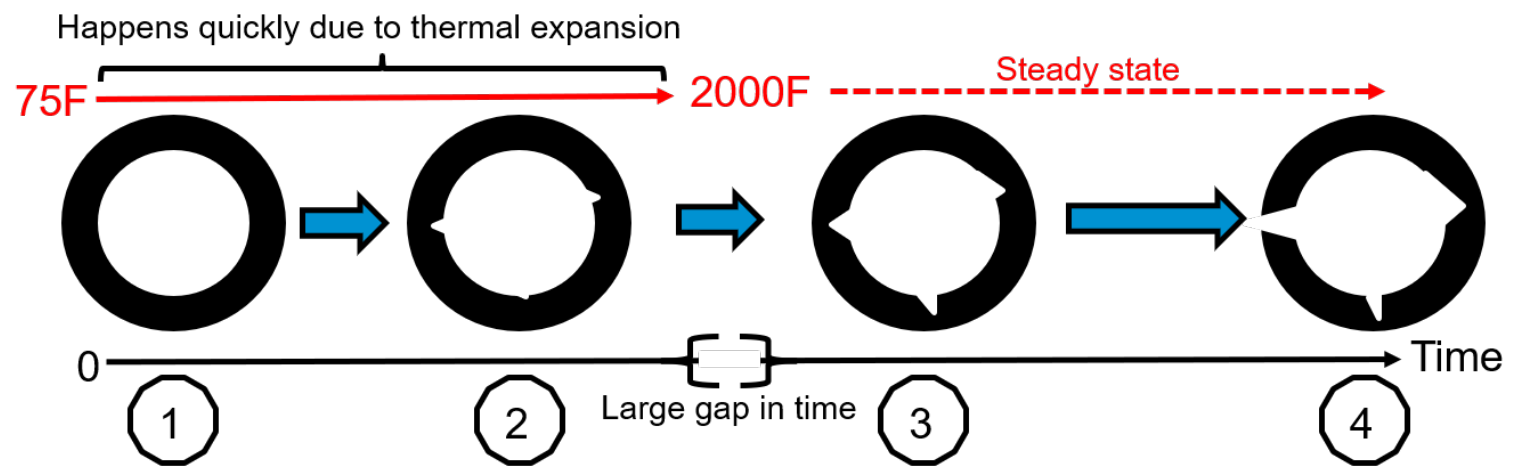

Figure 3.2: Visual Depiction of Sheath Failure Theory.

Figure 3.2 represents a visual depiction of the thermocouple failure over time when considering structural dynamic effects. The figure depicts the sheath as the thick black circle. Starting on the far left side with the sheath labeled as 1, this represents the sheath at room temperature to low-temperature values. Keep in mind that the image depicted is of a perfectly round and polished surface sheath. It is highly likely that this would not be the case in a real-world application. The effect of which would expedite the process seen in Fig. 3.1. Then, moving to the right with the probe labeled as number 2 , this represents the initial case has increased the temperature to the point where small cracks form along the inside of the sheath which continue to grow as the temperature increases. Now to probe image number 3 and after some gap in time, the temperature has finally reached a steady-state and the cracks within the sheath have grown compared to when they first formed. Finally, the case at probe image number 4 has been achieved after being exposed to a constant high temperature and increasing vibrational effects. A crack has propagated through the entire sheath. At this point, the sheath has failed and it is likely that after this point the flow would rip the thermocouple apart and data gathering capability would be lost. Based on this proposed theory, future work must look into these kinds of dynamic failure modes. 


\section{Bibliography}

[1] Vincent, T. G., "Total Temperature Probe Performance for Subsonic Flows using Mixed Fidelity Modeling," Ph.D. Dissertation, Virginia Tech 2019.

[2] Schneider, A.J. "Computational modeling of total temperature probes." MS Thesis. Virginia Tech. 2015.

[3] Englerth, S.T., An Experimental Conduction Error Calibration Procedure for Cooled Total Temperature Probes, Thesis, Virginia Polytechnic Institute and State University, 2015.

[4] Eric Nicholas Rolfe. "Impact of Total Temperature Probe Geometry on Sensor Flow and Heat Transfer," MS Thesis, 2017.

[5] Reardon, J.P., Schetz, J.A. and Lowe, K.T., "Computational Modelling of TotalTemperature Probes," J. Thermophysics and Heat Transfer, vol. 31, no. 3, pp. 609-620, 2017.

[6] Quickel, R., "Mount Interference and Flow Angle Impacts on Unshielded Total Temperature Probes," MS Thesis, 2019 